\title{
Toward an international practical pressure scale: A proposal for an IPPS ruby gauge (IPPS-Ruby2020)
}

\author{
Guoyin Shen ${ }^{1}$, Yanbin Wang ${ }^{2}$, Agnes Dewaele ${ }^{3}$, Christine $\mathrm{Wu}^{4}$, Dayne E. Fratanduono ${ }^{4}$, Jon Eggert ${ }^{4}$, \\ Stefan Klotz ${ }^{5}$, Kamil F. Dziubek ${ }^{6}$, Paul Loubeyre ${ }^{3}$, Oleg V. Fat'yanov ${ }^{7}$, Paul D. Asimow ${ }^{8}$, Tsutomu \\ Mashimo $^{9}$, Renata M. M. Wentzcovitch ${ }^{10}$, and other members of the IPPS task group* \\ 1. HPCAT, X-ray Science Division, Argonne National Laboratory, Argonne, IL 60439, USA \\ 2. Center for Advanced Radiation Sources, University of Chicago, Argonne, IL 60439, USA \\ 3. CEA, DAM, DIF, 91297 Arpajon, France; and Université Paris-Saclay, CEA, Laboratoire Matière en Conditions Extrêmes, \\ 91680 Bruyères-le-Châtel, France \\ 4. Physics Division, Lawrence Livermore National Laboratory, Livermore, CA 94550, USA \\ 5. Sorbonne Université, IMPMC, UMR 7590, 75252 Paris, France \\ 6. LENS, European Laboratory for Non-linear Spectroscopy, 50019 Sesto Fiorentino, Italy \\ 7. QRate, 9 Donskoi 2nd Passage, Moscow 115419, Russia, also at Russian Quantum Center, 30 Bolshoi Blvd., Skolkovo \\ 121205, Russia \\ 8. Division of Geological and Planetary Sciences, California Institute of Technology, Pasadena, CA 91125, USA \\ 9. Shock Wave and Condensed Matter Research Center, Kumamoto University, Kumamoto 860-8555, Japan \\ 10. Applied Physics and Applied Mathematics, Columbia University, New York, NY 10027, USA
}

Abstract: At the $26^{\text {th }}$ AIRAPT conference in 2017, a task group was formed to work on an International Practical Pressure Scale (IPPS). This report summarizes the activities of the task group toward an IPPS ruby gauge. We have selected three different approaches to establishing the relation between pressure $(P)$ and ruby R1-line shift $(\Delta \lambda)$ with three groups of optimal reference materials for applying these approaches. Using a polynomial form of the second order, the recommended ruby gauge (referred as Ruby2020) is expressed by: $P[G P a]=$ $1.87( \pm 0.01) \times 10^{3}\left(\frac{\Delta \lambda}{\lambda_{0}}\right)\left[1+5.63( \pm 0.03)\left(\frac{\Delta \lambda}{\lambda_{0}}\right)\right]$, where $\lambda_{0}$ is the wavelength of the R1-line near $694.25 \mathrm{~nm}$ at ambient condition. In June of 2020, the Executive Committee of AIRAPT endorsed the proposed Ruby2020. We encourage high-pressure practitioners to utilize Ruby2020 within its applicable pressure range (up to $150 \mathrm{GPa}$ ), so that pressure data can be directly compared across laboratories and amended consistently as better scales emerge in the future.

\footnotetext{
*Other members of the IPPS task group between 2017-2019: Jay Bass, Department of Geology, University of Illinois, Urbana-Champaign, IL, USA; Yan Bi, HPSTAR, Beijing Laboratory, Beijing, China; Duanwei He, College of Physical Sciences and Technology, Sichuan University, Chengdu, China; Konstantin V. Khishchenko, Joint Institute for High Temperatures RAS, Izhorskaya 13 Bldg 2, Moscow 125412, Russia; Kurt Leinenweber, School of Molecular Sciences, Arizona State University, Tempe, AR, USA; Baosheng Li, Mineral Physics Institute, Stony Brook University, Stony Brook, NY, USA; Mohamed Mezouar, European Synchrotron Radiation Facility, Grenoble, France; Takeshi Sakai and Taku Tsuchiya, Geodynamics Research Center, Ehime University, Ehime, Japan; Katsuya Shimizu, Center for Science and Technology under Extreme Conditions, Osaka University, Suita, Osaka 565-0871, Japan; Daisuke Yamazaki, Institute for Planetary Materials, Okayama University, Missasa, Japan.
} 


\section{Introduction}

The first AIRAPT task group on the International Practical Pressure Scale (IPPS) was created at the $6^{\text {th }}$ AIRAPT Conference in 1977. From 1982 to 1986, the group recommended a ruby pressure scale, the Decker equation of state for $\mathrm{NaCl}$, and a set of pressure reference points for opaque presses be used as practical pressure reference standards [1, 2]. A decade later, the first international pressure calibration workshop was held in Japan in 1997, with a proposal of several reference points for pressure reference standards $[3,4]$. Since then, several major developments have occurred in high-pressure technology. Static pressure has been expanded to the multi-Mbar (1 Mbar=100 GPa) range [5-7], demanding revision on existing pressure standards or new pressure scales. In situ X-ray probes allow for pressure determination from measured unit-cell volumes of materials based on the knowledge of their equations of state (EOSs) [8-10], providing crosschecks of the previously established pressure gauges. Techniques for in-situ elasticity measurements (e.g., Brillouin scattering and ultrasonic techniques) have emerged to yield pressure information based on measured bulk modulus as a function of unitcell volume $[11,12]$. Progress in shock compression experiments has led to improved precision on velocity measurements and constraints on Grüneisen parameters [13, 14]. Shockless compression (more commonly referred to as ramp compression) provides access to regimes of pressure-temperature space inaccessible by single shock wave experiments, resulting in colder, denser states of matter closer to the room temperature isotherms than shock experiments [1518]. The ramp compression technique offers knowledge of the EOS of solid materials at multiMbar pressures. Lastly, theoretical calculations have become increasingly accurate tools for predicting the EOS of materials [19-22].

These developments call for a new IPPS. At the $26^{\text {th }}$ AIRAPT conference in 2017 , an IPPS task group was formed to work on a pressure scale that is (1) practical, so that an individual laboratory can use the scale to measure pressure without having access to large facilities such as synchrotron beamlines and can compare pressures derived from the new scale with those determined using other techniques; (2) amendable, so that the scale can be refined and improved with future developments; and (3) versatile, with various gauges covering different pressure ranges including low (<12 GPa), medium (12-150 GPa), and ultra-high (>150 GPa) pressure regions, and continuously applicable from low to high temperature.

One current focus of the task group is an IPPS ruby gauge at room temperature, covering a pressure range up to $150 \mathrm{GPa}$. A proposal for such a ruby gauge was presented at the $27^{\text {th }}$ AIRAPT conference in 2019 and has been widely discussed among scientists worldwide. Even though there are on-going discussions regarding some of the details, a general consensus has emerged. The proposal presented here is a summary of our discussions on an IPPS ruby gauge Ruby2020, which was sent to the Executive Committee of AIRAPT as a report of the task group. The executive committee endorsed the Ruby2020 gauge in June of 2020. 


\section{Calibrating pressure against ruby fluorescence shift}

The ruby fluorescence method has been widely used for decades for pressure measurement in the diamond anvil cell (DAC) and other optically transparent pressure cells [23-25]. A ruby pressure gauge is a quantitative relation between applied pressure and wavelength shift (or frequency change) of R-line emission from stimulated ruby. The R-line emission of ruby involves two components (R1- and R2-lines) having similar responses to hydrostatic pressure but displaying different behavior under nonhydrostatic conditions [26]. Here we only consider the more intense R1-line in pressure calibration. At pressures below $20 \mathrm{GPa}$, the shift of the R1line may be approximated as linear [24]. At higher pressures, however, the R1-line shift becomes significantly nonlinear. This nonlinear behavior is the main concern in developing an accurate extended ruby gauge and has been extensively studied in recent years. Developments in high-pressure technology and integration with synchrotron radiation techniques have enabled diffraction measurements with intense, well-focused X-ray beams on small samples in helium-loaded DACs at pressures extending well beyond $100 \mathrm{GPa}$. Helium is widely held to be the most hydrostatic among available pressure medium choices [27]. Simultaneous loading of small ruby spheres in the same cells has led to high precision data on the relation between specific volume $(V)$ of many materials and the ruby R-line shift $(\Delta \lambda)$ at high pressure [8-10]. Based on recently obtained $V$ - $\Delta \lambda$ relations, several ruby pressure gauges have been proposed $[10,16,28-40]$; discrepancies among these recent ruby gauges are typically within $3 \%$ for pressures up to $150 \mathrm{GPa}$. However, various approximations underlie each of these ruby gauges. So far, it appears that no single gauge has emerged as a dominant standard in the high-pressure community. A call for a "unified" ruby gauge was discussed at each of the last two AIRAPT conferences.

Here we recommend a ruby gauge (Ruby2020), with the following considerations:

- the Ruby2020 gauge is based on data without prior assumptions about pressure;

- it is easily traceable and amendable in the future as better data become available;

- it is calibrated against representative EOS data for ionic, covalent, and metallic materials; and

- it covers the pressure range up to $150 \mathrm{GPa}$ at room temperature for which $V$ - $\Delta \lambda$ data are available with helium as pressure medium.

Based on the above considerations, we have selected three different approaches to establishing the relation between pressure $(P)$ and ruby R1-line shift $(\Delta \lambda)$ with three groups of optimal reference materials for applying these approaches: (1) molybdenum (Mo), and copper (Cu), (2) periclase ( $\mathrm{MgO})$, and (3) diamond. The first approach involves metals whose $P-V$ relations are constrained by dynamic data, where pressure and density are derived directly from the conservation equations. The MgO calibration is based on in-situ elasticity measurements that use Brillouin scattering to establish a relationship between the adiabatic bulk modulus $\left(K_{S}\right)$ and the specific volume, from which pressure can be determined by integrating the isothermal bulk modulus $\left(K_{T}\right)$ from the reference volume to the volume of 
interest. The diamond calibration is based on knowledge of the EOS and constraints on the elastic properties with $K_{T}$ at ambient pressure $\left(K_{T O}\right)$ constrained by experimental measurements and its pressure derivative $\left(K_{T O}{ }^{\prime}\right)$ constrained by computation simulations.

We use these $P-V$ relations, without prior assumptions about pressure or dependence on other pressure scales, to establish $P$ - $\Delta \lambda$ calibrations by converting $V$ to $\Delta \lambda$ using the measured $V-\Delta \lambda$ relations from simultaneous ruby fluorescence and synchrotron-based diffraction results in helium-loaded DACs. The conversion procedure implies an assumption of negligible pressure differences between the sample and the nearby small ruby spheres within the DAC chambers. Therefore, we select the $V$ - $\Delta \lambda$ data where well-focused small-spot synchrotron $x$-rays were used to determine volume with helium as the least non-hydrostatic and the most spatially homogeneous pressure medium $[9,12,30]$.

\subsection{Mo and $\mathrm{Cu}$}

To estimate isothermal equations of state from dynamic compression, we select two metals (Mo and $\mathrm{Cu}$ ) as calibrants. The main arguments behind this selection include: (1) both Mo and Cu were originally used as primary calibrants in the widely used Mao-78 and Mao-86 ruby gauges [25, 41]; (2) there are a significant number of absolute, symmetric impact Hugoniot data up to more than $300 \mathrm{GPa}$ for both Mo and $\mathrm{Cu}$; (3) in reduced-shockwave-analysis, both Mo and $\mathrm{Cu}$ show relatively small thermal contributions up to $150 \mathrm{GPa}$ (see Fig. S1 in the Supplemental Materials); and (4) a new study of ramp compression in $\mathrm{Cu}$ [42] provides an isentrope that, reduced to a $298 \mathrm{~K}$-isotherm, agrees with the reduced Hugoniot data [43-45] within $1 \%$ up to $150 \mathrm{GPa}$.

For Mo, four sources of room-temperature isotherms from Hugoniot data [45-48] are in mutual agreement within $1 \%$ up to $250 \mathrm{GPa}$. The 293K-isotherm from Al'tshuler et al [43] is slightly stiffer at pressures above $150 \mathrm{GPa}$ (see Table S1, Fig. S2). Within the pressure range up to $150 \mathrm{GPa}$, the combined data from all five sources $[43,45-48]$ can be interpolated accurately by using an EOS. We use the EOS-AP2 form proposed by Holzapfel [49] to represent the consensus isotherm for Mo:

$$
P(x)=3 K_{T 0} \frac{1-x}{x^{5}}\left[1+c_{2} x(1-x)\right] \exp \left[c_{0}(1-x)\right],
$$

where $x=\left(V / V_{0}\right)^{1 / 3}, c_{0}=-\ln \left(3 K_{T O} / P_{F G O}\right), c_{2}=(3 / 2)\left(K_{T O}{ }^{\prime}-3\right)-c_{0}, P_{F G O}=1003.6\left(Z / V_{0}\right)^{5 / 3} \mathrm{GPa}, V_{0}$ is the molar volume in $\mathrm{cm}^{3} / \mathrm{mole}$, and $Z$ is the total number of electrons per structural unit (for Mo, it is the atomic number). For Mo, the value of $K_{T O}$ is constrained to be $260 \mathrm{GPa}$ by the experimental data at ambient condition [50-52]. The best fit to the room-temperature isotherms gives $K_{T O}{ }^{\prime}$ of $4.00(1)$, with a small root-mean-square (RMS) of $0.5 \mathrm{GPa}$ up to $150 \mathrm{GPa}$ (Fig. S2). The RMS value reflects the small errors from the selection of the EOS form (Eq. 1) and the scatter among data from the literature.

For $\mathrm{Cu}$, reduced Hugoniot data from two experimental groups $[46,53]$ and two computational groups $[38,45]$ are in general agreement within $1 \%$ up to $150 \mathrm{GPa}$ (Fig. S3). A 
study by ramp-compression [16] shows a slightly stiffer isotherm at $293 \mathrm{~K}$ than the reduced Hugoniot data at pressures up to $150 \mathrm{GPa}$. Very recently, a new ramp compression study [42] gives a $298 \mathrm{~K}$-isotherm that is consistent with the reduced Hugoniot data within $1 \%$ up to 150 GPa. We therefore use the newer ramp-compression data (Table 1 in [42]) for Cu in our analysis. The small temperature difference $(293 \mathrm{~K}, 298 \mathrm{~K})$ in representing room temperature is ignored.

The $V-\Delta \lambda$ data for Mo and $\mathrm{Cu}$ are from [30] and [29], respectively, where the specific volumes were measured using synchrotron radiation with adjacent samples and ruby spheres in helium environments. Conversion of the specific volumes to pressures using the dynamic compression-derived room temperature isotherms yields $P-\Delta \lambda$ relations for $\mathrm{Mo}$ and $\mathrm{Cu}$, plotted in Fig. 1 and numerically tabulated in Table S2.

Major sources of uncertainty in the reduced Hugoniot data include (1) precision in the Hugoniot measurements, (2) accuracy in accounting for thermal pressure contribution that requires a Grüneisen parameter model and specific heat estimate, and (3) material strength effects. The fit to the Mo Hugoniot data [45-48] in the $U_{s}-U_{p}$ plane (where $U_{s}$ is the shock velocity and $U_{p}$ is the material velocity) typically yields deviations between the fit and the data of less than $\pm 1 \%$ in pressure. The maximum uncertainty in the pressure on the roomtemperature isotherm at $150 \mathrm{GPa}$ from the thermal correction, primarily due to the uncertainty in Grüneisen parameter, is $\pm 0.7 \%$ for Mo (Fig. S1). Nonhydrostatic stress during dynamic compression needs to be corrected for the material yield strength $(Y)$ to obtain hydrostatic stress [54]. The yield strength increases with shock stress for Mo $[55,56]$ in the pressure range up to $150 \mathrm{GPa}$. Assuming a linear increase in $Y$ with stress, a maximum pressure correction at $150 \mathrm{GPa}$ is found to be about $-2.3 \mathrm{GPa}$ (or $-1.5 \%$ ) for Mo [48]. Convolving all the above uncertainties gives a total uncertainty in the pressure of $\pm 1.9 \%$ on the room-temperature isotherm of Mo.

Major sources of uncertainty in the reduced isotherm from ramp compression are similar to reduced Hugoniot data. The corrections include (1) experimental precision in determining the Lagrangian sound velocity versus particle velocity and target metrology, (2) accuracy in the model that relates pressures on the ramp compression path and on the isotherm, which involves the high-pressure Grüneisen parameter and estimates of heating by plastic deformation, and (3) material's strength effects. Since both Hugoniot and ramp compression require similar corrections to reduce to the isotherm, authors in [42] applied an iterative procedure to self-consistently solve for the pressure along the isotherm using all available dynamic data including those from shock and ramp compression. In this way, the uncertainty in the Grüneisen parameter for Cu propagates to a $\pm 0.2 \%$ uncertainty in the pressure along the isentrope $[16,42]$. The uncertainties in pressure arising from the yield strength and heating due to plastic deformation are both less than $\pm 0.5 \%$ in the concerned pressure range of interest [42]. Using the fitted upper and lower bounds, one arrives at a total uncertainty of $\pm 2 \%$ in the pressure on the $298 \mathrm{k}$-isotherm up to $150 \mathrm{GPa}$ for $\mathrm{Cu}$ [42]. The $298 \mathrm{~K}$-isotherm combining shock 
and ramp compression is consistent with the reduced Hugoniot data $[38,43,46,57]$ within $1 \%$ up to $150 \mathrm{GPa}$. The uncertainty of the reduced Hugoniot data for $\mathrm{Cu}$ can be estimated, similar to that for Mo, by the precision in the dynamic measurements $( \pm 1 \%)$, the maximum uncertainty at $150 \mathrm{GPa}$ from the thermal correction $( \pm 1.7 \%$ ) (Fig. S1), and the materials' strength effects ($1.5 \%)$ [41], yielding a total uncertainty of $\pm 2.5 \%$ for $\mathrm{Cu}$.

Turning to the $V-\Delta \lambda$ relations, errors in individual $V$ and $\lambda$ measurements are both negligibly small $(<0.1 \%)$. The main source of error is the nonhydrostaticity of helium medium above $\sim 30$ GPa [58]. Uniaxial differential stress can reach $\sim 1 \mathrm{GPa}$ at pressures above $100 \mathrm{GPa}$ [58], which suggests potential differences of $\sim \pm 1 \%$ between the sample (where $V$ is measured) and the ruby spheres (where $\lambda$ is measured). After considering the uncertainty in determining the $V-\Delta \lambda$ relations for $\mathrm{Mo}$ and $\mathrm{Cu}( \pm 1 \%)$, the propagated uncertainty in pressure at a given $\Delta \lambda$ on the room-temperature isotherm is estimated to be $\pm 2.0 \%$ for Mo and $\pm 2.7 \%$ for $\mathrm{Cu}$, respectively.

\section{2. $\mathrm{MgO}$}

Zha et al [12] loaded an MgO single crystal into a DAC with helium as pressure medium and measured elastic wave velocities by Brillouin scattering, specific volume by $x$-ray diffraction, and ruby fluorescence shifts at pressures up to $55 \mathrm{GPa}$. After correction from the adiabatic to the isothermal bulk modulus, this set of data allows pressure values to be derived directly by integrating the bulk modulus data as a function of volume. The resultant pressure can then be used to calibrate a $P-\Delta \lambda$ relation for $\mathrm{MgO}$ with the $\mathrm{R} 1$-line shifts of ruby grains measured in the same sample chamber. The results are shown in Fig. 1, with numerical results tabulated in Table S2.

In principle, this approach is independent of any model assumptions. A claim of $\pm 1 \%$ accuracy is reported for the $P$ - $\Delta \lambda$ relation up to $55 \mathrm{GPa}$ [12]. However, due to the limited experimental points in the covered pressure range, a presumed EOS was used to interpolate the bulk modulus and the volume data under compression. Because pressure is derived by integrating bulk modulus as a function of volume, the selection of EOS form may cause some uncertainties in the derived pressure. Other possible errors include assumptions about the values and functional forms of the thermal expansion and Grüneisen coefficients used to correct adiabatic to isothermal bulk moduli (a $2 \%$ total correction with uncertainty presumably much less than that), summation of errors during integration, and potential misalignment of the MgO crystal in the Brillouin measurements. Due to the lack of details in the reported data, it is difficult to estimate these systematic errors. Another issue is the limited pressure range (0$55 \mathrm{GPa}$ ) covered by this approach. To better constrain the nonlinear behavior of ruby shift up to $150 \mathrm{GPa}$, we should include other calibrants.

\subsection{Diamond}

When the change of volume of a solid due to compression is small $\left(\Delta V / V_{0}<15 \%\right)$, any appropriate EOS form (such as the commonly used the third-order Birch-Murnaghan, Vinet, or 
Holzapfel-AP2 forms) can reasonably represent its $P-V$ relation, with insignificant difference in the result due to choice of EOS form. Hence, it is possible to assume an EOS form, with parameters $\left(K_{T O}, K_{T O^{\prime}}\right)$ constrained by ultrasonic and Brillouin measurements at ambient and low pressures and/or by theoretical simulations, to describe the compression behavior of an incompressible solid without suffering from large uncertainties in pressure. As diamond has the highest known bulk modulus, its volume change upon compression is smaller than any other material one might choose. This makes diamond an ideal material to use in applying EOS forms to represent the $P-V$ relation up to $150 \mathrm{GPa}$, a range for which the ratio of pressure over the bulk modulus of diamond $\left[P / K_{T}(P)\right]$ is $<0.15$. For example, when the three aforementioned EOS forms are applied, the differences in pressure are less than $1 \mathrm{GPa}$ at $\sim 150 \mathrm{GPa}$ (or $<0.7 \%$ ), and less than $2 \mathrm{GPa}$ at $\sim 200 \mathrm{GPa}$ (or $<1 \%$ ).

The adiabatic bulk modulus of diamond at ambient pressure $\left(K_{s o}\right)$ has been measured using ultrasonic and Brillouin scattering techniques [59-61]. The $K_{s o}$ values range from $442 \mathrm{GPa}$ to $444.8 \mathrm{GPa}$, with a median and interquartile range of $443.4 \pm 1.4 \mathrm{GPa}$. Using the available data of Grüneisen parameter [9] and thermal expansion coefficient [39] for diamond, the correction from $K_{S O}$ to $K_{T O}$ is small, yielding a $K_{T O}$ of $443.3 \pm 1.4 \mathrm{GPa}$.

The pressure derivative of adiabatic bulk modulus $\left(K_{s o}\right)$ for diamond has been experimentally determined to be $4.0 \pm 0.7$ from ultrasonic measurements up to $0.12 \mathrm{GPa}$ [60]. The reported uncertainty, estimated from "measured slopes in error by $10 \%$ ", can lead to an uncertainty of $\sim 10 \%$ in pressure at $150 \mathrm{GPa}$, which is too large for calibrating a pressure gauge. On the other hand, due to its simple and highly symmetric structure and simple electronic configuration, diamond is an ideal candidate for performing quantum mechanical calculations. Typically, calculated volume, pressure, and bulk modulus depend on the approximation used for the exchange-correlation term in density functional theory. However, as a volume dependent parameter, $K_{T O}$ ' for diamond is found to be insensitive to theoretical approximations $[19,62,63]$, with its values falling in a narrow range of $3.6-3.8$, with a median value and interquartile range of $3.7 \pm 0.1$. If we take this \pm 0.1 range as the uncertainty in $K_{T O}{ }^{\prime}$, it corresponds to a maximum uncertainty of $\pm 1.1 \%$ in pressure up to $150 \mathrm{GPa}$.

Among the EOS forms of the third-order Birch-Murnaghan, Vinet, Holzapfel-AP2, we find that, at a given compression ratio $V / V_{0}$ for diamond, the pressure value obtained from the EOSAP2 lies in between those from the third order Birch-Murnaghan EOS and from the Vinet EOS up to $150 \mathrm{GPa}$. Hence, we use Holzapfel's AP2 form [49] (Eq. 1) to represent the $P$-V relation of diamond. Applying $K_{T O}$ of $443.3 \mathrm{GPa}$ and $K_{T O}{ }^{\prime}$ of 3.7 , we have $c_{0}=0.6583$, and $c_{2}=0.3917$ for the parameters in Eq. (1). Combining the $P-V$ relation with the experimentally determined $V-\Delta \lambda$ data for diamond [9], we obtain a calibration of the $P-\Delta \lambda$ relation for diamond, plotted in Fig. 1 and tabulated in Table S2.

Major sources of error in the $P-V$ relation of diamond include the selection of EOS form $( \pm 0.7 \%)$, scatter of the $K_{T O}$ estimates $( \pm 0.3 \%)$, and the uncertainty in $K_{T O}{ }^{\prime}( \pm 1 \%)$. Even though 
individual computational model results typically constrain $K_{T O}{ }^{\prime}$ within $\pm 0.05[19,62,63]$, we double the range and use $K_{T O^{\prime}}=3.7 \pm 0.1$ to cover the differences in fitted values among published studies. Convolving these three sources of error, the uncertainty in pressure in the EOS of diamond is estimated to be $\pm 1.3 \%$. Considering the uncertainty of $\pm 1 \%$ in the $V-\Delta \lambda$ relation, the overall uncertainty in pressure in the $P-\Delta \lambda$ calibration is $\pm 1.6 \%$. A precise experimental $K_{T O}$ ' value for diamond is urgently needed to validate the theoretically calculated value adopted here. Such experiments can be performed over a modest pressure range in a hydrostatic helium medium below its solidification pressure (<12 GPa).

\section{An IPPS ruby gauge - Ruby2020}

The above $P$ - $\Delta \lambda$ relations -- from two metals ( $\mathrm{Mo}$ and $\mathrm{Cu}$ ), $\mathrm{MgO}$, and diamond -- represent three approaches with different sources of data and different underlying approximations. Yet, it is shown that their $P$ - $\Delta \lambda$ plots converge to a single nonlinear relation (Fig. 1 ). We use a polynomial form of the second order to fit the nonlinear behavior.

$$
P=A\left(\frac{\Delta \lambda}{\lambda_{0}}\right)\left[1+B\left(\frac{\Delta \lambda}{\lambda_{0}}\right)\right]
$$

where $P$ is the pressure in $\mathrm{GPa}, \lambda_{0}$ is the wavelength of the $\mathrm{R} 1$ emission line of ruby observed near $694.25 \mathrm{~nm}$ at ambient condition, $\Delta \lambda\left(=\lambda_{P}-\lambda_{0}\right)$ is the shift in wavelength at $P\left(\lambda_{P}\right)$ relative to $\lambda_{0}$, the parameter $A$ is the initial slope at 1 bar, and the parameter $B$ represents the nonlinear term in the polynomial form. 


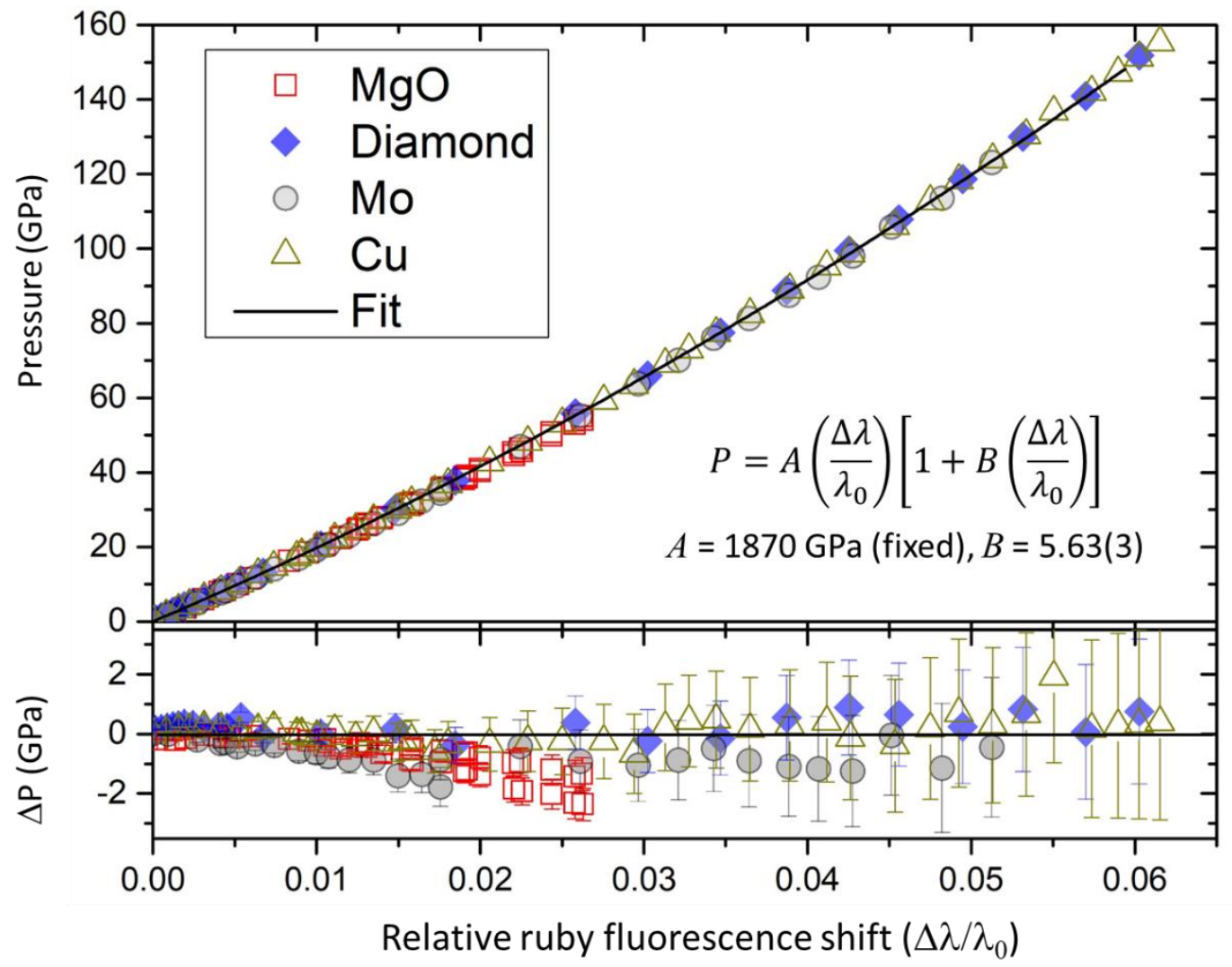

Figure 1. The relation of pressure versus ruby R1-line shift for two metals (Mo and $\mathrm{Cu}$ ), $\mathrm{MgO}$, and diamond. For Mo and $\mathrm{Cu}$, the $V-\Delta \lambda$ data are from [30] and [29], respectively. For MgO, the $P-\Delta \lambda$ data are directly from [12]. For diamond, the $V-\Delta \lambda$ data are from [9]. The line is the fit in the form of $A\left(\Delta \lambda / \lambda_{0}\right)\left[1+B\left(\Delta \lambda / \lambda_{0}\right)\right]$ with $A=1870 \mathrm{GPa}$ (fixed) and $B=5.63(3)$. The fit gives a root-mean-square value of $0.7 \mathrm{GPa}$ in the pressure range up to $150 \mathrm{GPa}$.

We first constrain the initial slope $(A)$ from the low-pressure data by back-extrapolating the $P$ - $\Delta \lambda$ data points to 1 bar using Eq. (2). Note that, because of nonlinearity, if the $A$-values are obtained from a linear approximation over a large pressure range, they can be overestimated. Here, we select a pressure range of $0-12 \mathrm{GPa}$, where the pressure medium (helium) remains liquid. A back-extrapolation of all the data (Mo, $\mathrm{Cu}, \mathrm{MgO}$, diamond) below $12 \mathrm{GPa}$ according to Eq. (2) gives $A=1.87(1) \times 10^{3} \mathrm{GPa}$, with the $B$ value fixed at 5.5. Changing $B$ by \pm 0.5 has negligible effect on the resultant $A$ values (within $\pm 2 \mathrm{GPa}$ ). The obtained $A$-value may be compared with those estimated by using the low-pressure data of the ruby fluorescence shifts against specific volumes of $\mathrm{NaCl}[8,24]$. By converting the $V-\Delta \lambda$ data to $P-\Delta \lambda$ relations using the EOS of $\mathrm{NaCl}[21,22,64]$, we obtain $A=1890$ (5) GPa from Brown's EOS for $\mathrm{NaCl}$ [21], $A=1848(6)$ GPa from Marcondes and Wentzcovitch's EOS [22], and $A=1833(4) \mathrm{GPa}$ from Decker's EOS [64], all with $B$ fixed at 5.5 and a similar pressure range of $0-12 \mathrm{GPa}$. Our constrained $A$-value, $1.87(1) \times 10^{3} \mathrm{GPa}$, is generally consistent with those from $\mathrm{NaCl}$ data within the uncertainties in the EOS from different sources. 
We then use the high-pressure data to constrain the nonlinearity (i.e. $B$ ) in the $P-\Delta \lambda$ relation by fixing the $A$-value at $1870 \mathrm{GPa}$. Fitting all the $P-\Delta \lambda$ data points for the two metals (Mo and $\mathrm{Cu}$ ), $\mathrm{MgO}$, and diamond to Eq. (2) results in $B=5.63(3)$ with a RMS value of $0.7 \mathrm{GPa}$ in the pressure range up to $150 \mathrm{GPa}$. In the fitting procedure, the data from the three methods we have applied are equally weighted, i.e., $1 / 3$ is weighted to the two metals' data points (1/6 for Mo, $1 / 6$ for $\mathrm{Cu}$ ), $1 / 3$ to $\mathrm{MgO}$ data points, and $1 / 3$ to diamond data points, respectively.

In summary, the $P$ - $\Delta \lambda$ calibrations derived from analysis of two metals ( $M o$ and $\mathrm{Cu}$ ), $\mathrm{MgO}$, and diamond can be represented by Eq. (2) as

$$
P[G P a]=1.87( \pm 0.01) \times 10^{3}\left(\frac{\Delta \lambda}{\lambda_{0}}\right)\left[1+5.63( \pm 0.03)\left(\frac{\Delta \lambda}{\lambda_{0}}\right)\right]
$$

with a RMS value of $0.7 \mathrm{GPa}$. A maximum uncertainty in pressure in the pressure range up to $150 \mathrm{GPa}$ is estimated to be $\pm 2.5 \%$. This is our recommended Ruby 2020 gauge, which was endorsed by the Executive Committee of AIRAPT. We encourage high-pressure practitioners to utilize Ruby2020 within its applicable pressure range, so that pressure data can be directly compared across laboratories and amended consistently as better scales emerge in the future.

\section{A few notes on the Ruby2020 gauge}

\subsection{Comparison with published ruby gauges}

At pressures up to $50 \mathrm{GPa}$, Ruby2020 is in general agreement with all the published gauges within the gauge uncertainty of $2.5 \%$ (Fig. 2). At higher pressures, Ruby2020 tends to be in agreement with more recently published gauges. It is consistent with the gauges of Dewaele et al. (2008) [30], Dorogokupets and Oganov (2007) [65], and Chijioke et al. (2005) [44] within an uncertainty range of $1 \%$. If the uncertainty range extends to $2.5 \%$, more published gauges (Kunc et al. (2004) [35], Jacobsen et al. (2008) [10], Syassen (2008) [37], Sokolova et al. (2013) [40], and Holzapfel (2005) [39]) are within the range. However, we note that Ruby2020 lies toward the lower bound of the recently published gauges (Fig. 2). This may be viewed as a coincidence, because calibrating a gauge can be affected by many factors, including the selection of calibrants, the data sources used, the approaches to calibration, and even the analytical form such as Eq. 2 to represent the nonlinear behavior. We include a summary of published ruby gauges in the Supplemental Materials (Table S3). 


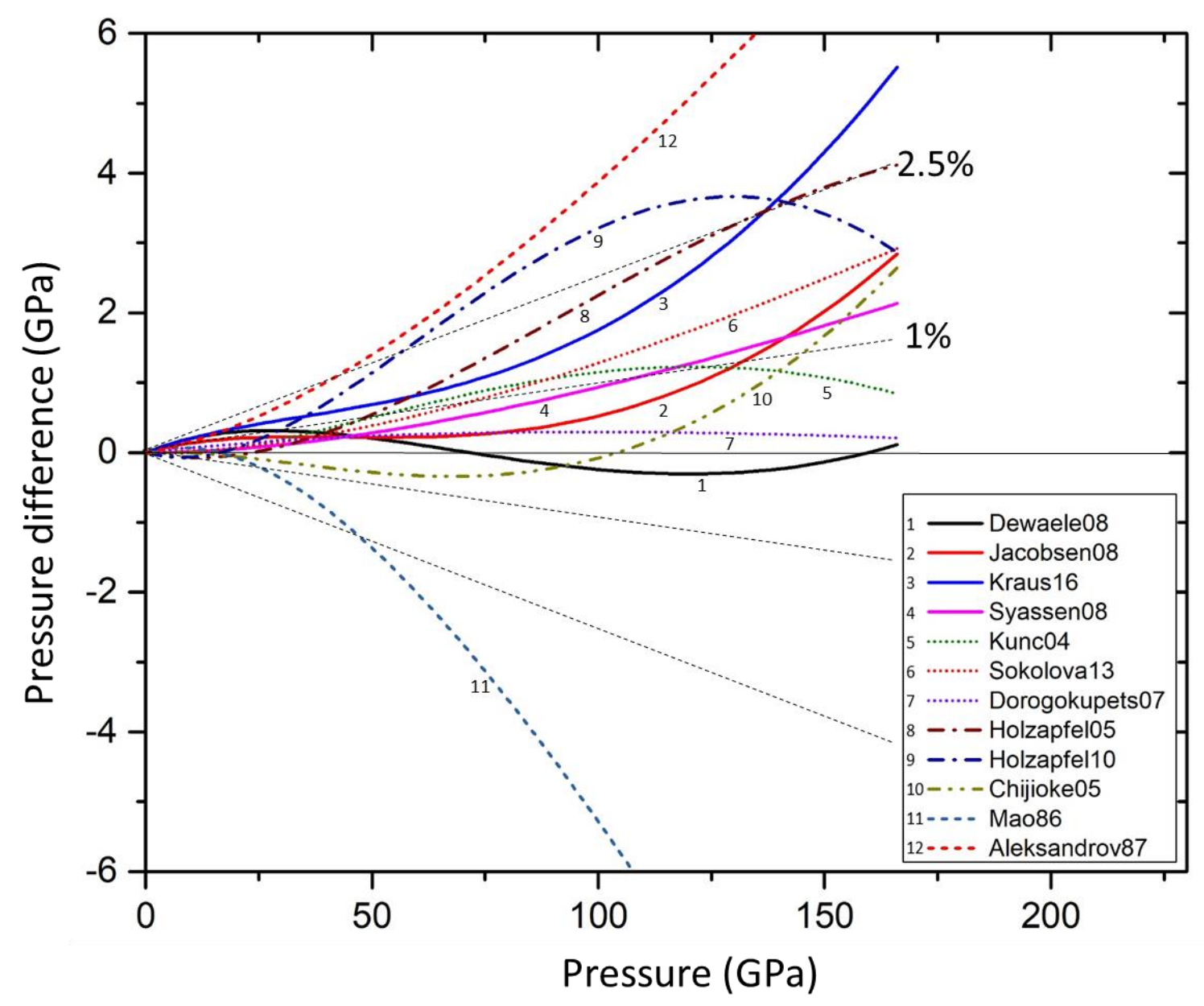

Figure 2. The pressure difference of recently published ruby gauges relative to the Ruby2020 gauge. The listed gauges are summarized in Table S3.

\subsection{Calibration at low pressures $(<12 \mathrm{GPa})$}

As shown in Fig. 3 for pressures below $12 \mathrm{GPa}$, Ruby2020 provides a calibration consistent with all the published gauges within a relative uncertainty of $\pm 2.5 \%$. Calibration at low pressures is largely affected by the initial slope $A$ in Eq. 2 . We note that, in publications where $A$-values were fixed and constrained from the linear coefficient averaged over a given pressure range, the initial slopes may be over-estimated and require a correction using the back-extrapolation procedure to 1 bar.

The precision in pressure using a ruby gauge can be $0.01 \mathrm{GPa}[37,66]$, estimated from the precision in ruby fluorescence measurements. With such a precision, we should be able to better constrain the initial slope $A$. The question is how we can assign a pressure at a given compression condition. As suggested by the previous AIRAPT IPPS task group [2], at pressures below $1.4 \mathrm{GPa}$, the melting curve of mercury, absolutely calibrated by primary standard piston gauges, may be used for pressure calibration. Other reference points with their pressures calibrated by primary piston gauges, such as the melting of $\mathrm{H}_{2} \mathrm{O}$ [66] and the I-II transition of $\mathrm{Bi}[20]$, may be used as well. Precisely constraining the initial slope $A$ from pressure calibrations based on piston gauges will be an important step to improve the accuracy of a ruby gauge, particularly at low pressures. 




Figure 3. The pressure difference of recently published ruby gauges relative to the Ruby2020 gauge at pressures below 12 GPa. The listed gauges are summarized in Table S3.

\subsection{Calibration at pressures over $150 \mathrm{GPa}$}

Ruby2020 is for a pressure range up to $150 \mathrm{GPa}$ at room temperature. Extrapolation to higher pressures may involve larger uncertainties in pressure determination. Furthermore, because ruby is structurally metastable at pressures above 80-100 GPa [67, 68], with its fluorescence intensity becoming weaker at higher pressures, alternative pressure gauges, such as Raman-based sensors of diamond [69, 70] and cBN [71, 72], may be used for pressure determinations above $150 \mathrm{GPa}$.

The key to proper pressure calibration above $150 \mathrm{GPa}$ is still accurate $P-V$ data. Considering the three approaches applied in developing Ruby2020, direct pressure determination from elastic wave velocities using Brillouin scattering and other acoustic techniques will become increasingly difficult at pressures above $150 \mathrm{GPa}$ and uncertainty from integration over a large pressure range may yield large accumulated errors. In the approach using an EOS to extrapolate compression behavior, an essential requirement is small relative volume change. At very high pressures, however, such extrapolation necessarily leads to increasing uncertainties in the $P-V$ relation. Therefore, at very high pressures $(>150$ $\mathrm{GPa}$ ), EOS data from direct measurements in dynamic compression may become the sole available source of experimental data, in particular ramp compression data that probe cool condensed materials, a method that is now routinely extended to the TPa range $[18,42]$. Finally, the increasing accuracy of 
computational simulations continues to support a paradigm shift in which computed EOS data may be treated as a primary source of information where experimental results are unavailable or unreliable.

\subsection{Deviatoric stress and pressure gradient}

Helium, with the highest freezing pressure of 12 GPa at room temperature among the rare gases, is widely used as pressure-transmitting medium in DAC experiments. Solid helium is generally viewed as quasi-hydrostatic above $12 \mathrm{GPa}[28,58]$. The deviatoric stress, due to the uniaxial loading geometry in a DAC and the strength of solid helium, becomes detectable above $30 \mathrm{GPa}$ [58], but is found to be small $(<1 \mathrm{GPa})$ even at pressures of 100-150 GPa $[27,28,58]$ when a sample remains embedded in heliummedium. Pressure gradients, which are limited by the strength of the pressure medium, are also small.

Note, however, that many factors can contribute to the stress distribution in DAC, such as sample preparation (initial size and shape of the sample chamber, particle size and dimension of the sample, amount of the pressure medium relative to the chamber size, etc), plastic deformation of the gasket, and stress conditions of the small samples at very high pressures. Such details of experimental conditions are specific to individual experiments, potentially having different degrees of uncertainty in stress conditions. Therefore, when Ruby2020 is applied for pressure determination, the stress condition of each individual experiment needs to be taken into account.

Deviatoric stress can be estimated by diffraction experiments, such as the obtained $\Gamma$-plots used in $[28,58]$. The ruby fluorescence technique is also applicable for evaluating non-hydrostaticity. The width of either R1- or R2-emission line is a measure of the stress inhomogeneity, while the R1-R2 splitting measures the deviatoric stress component. If a ruby sphere is under a uniform uniaxial stress condition, the R1- and R2-lines can remain sharp [26]. Thus, peak width alone cannot be used to address hydrostaticity in a DAC. Rather, a change in R1-R2 splitting may be an indication for the presence of deviatoric stress $[26,73]$.

Without pressure medium, or when pressure media other than helium are used, there may be significantly large deviatoric stresses and pressure gradients in DACs, resulting in large errors in pressure determination, no matter how accurate the pressure scale is. In such cases, one may use hightemperature annealing (both the sample and ruby) to reduce effects of uniaxial stress and pressure gradient.

\subsection{Some technical notes on ruby fluorescence measurement}

Ruby ( $\mathrm{Cr}$ doped $\alpha-\mathrm{Al}_{2} \mathrm{O}_{3}$ ) samples should be carefully chosen for reliable pressure measurements. A low $\mathrm{Cr}$ concentration $(<0.5 \%-\mathrm{Cr})$ ruby is most suitable. Both the intensity and the full width at half maximum (FWHM) of the R1 fluorescence line of ruby display a small increase with $\mathrm{Cr}$ concentration [74, 75]. A level of $0.3 \%-\mathrm{Cr}$ is found to be a good compromise between line width and intensity for the use of ruby as pressure marker [74]. Note that the use of annealed ruby spheres with released internal strain [74] is of considerable practical value, in particular for low pressure determinations.

The power of the excitation source should be kept low $(<10 \mathrm{~mW})$, in order to avoid local heating and broadening of the R1 fluorescence line. It is reported [74] that the effect of local heating is not significant when the power remains lower than $100 \mathrm{~mW}$, while the FWHM of the R1 line starts to increase with power above $10 \mathrm{~mW}$. 
A spectrometer with a pixel separation corresponding to a wavelength interval of $\sim 0.05 \mathrm{~nm}$ generally corresponds to a precision of $\sim 0.05 \mathrm{GPa}$ in pressure. For better precision in pressure measurement, a spectrometer with higher wavelength resolution is needed. However, precision is ultimately limited by the natural width of the R1 line at room temperature ( $0.6 \mathrm{~nm})$ [75] and aiming to achieve precision that greatly exceeds the accuracy of the calibration is probably a poor use of resources.

Since the pressure is calibrated against the shift of the $\mathrm{R} 1$ line relative to the position at ambient pressure, it is important to have a reference ruby sample held at ambient pressure but otherwise under conditions similar to the compressed ruby sample under study. For example, the reference ruby can be loaded in an empty space (no pressure) in a DAC, so that a similar level of power of the excitation source can be applied to the reference ruby and to the compressed ruby. The reference and pressure marker rubies should be selected from the same batch, so that their conditions ( $\mathrm{Cr}$ concentration, residual stress, etc.) are similar.

Acknowledgment: We thank the AIRAPT Executive Committee for the support of the IPPS task force activities. We also thank Drs. Steven Jacobsen and Thomas Duffy for useful discussions. GS acknowledges the support of HPCAT operations by Department of Energy (DOE)-NNSA's Office of Experimental Sciences. YW acknowledges the support of GeoSoilEnviroCARS, which is supported by the National Science Foundation - Earth Sciences (EAR - 1634415) and DOE-GeoSciences (DE-FG0294ER14466).

References:

[1] Bean, VE, Akimoto, S, Bell, PM, et al., in High Pressure in Research and Industry, C.M. Backman, T. Johannisson, and L. Tegner, Editors. 1982, Arkitektkopia Uppsala: Uppsala, Sweden. p. 144.

[2] Bean, VE, Akimoto, S, Bell, PM, et al., Another step toward an international practical pressure scale: 2nd AIRAPT IPPS task group report. Physica B+C, 1986. 139-140:52-54.

[3] Ito, E and Presnall, DC, Report on the first international pressure calibration workshop. The Review of High Pressure Science and Technology, 1998. 7:151-153.

[4] Ito, E, 2.08 - Theory and practice - Multianvil cells and high-pressure experimental methods, in Treatise on Geophysics, G. Schubert, Editor. 2007, Elsevier: Amsterdam. p. 197-230.

[5] Li, B, Ji, C, Yang, W, et al., Diamond anvil cell behavior up to 4 Mbar. Proc. Natl. Acad. Sci., 2018. 115(8):1713-1717.

[6] Dewaele, A, Loubeyre, P, Occelli, F, et al., Toroidal diamond anvil cell for detailed measurements under extreme static pressures. Nat. Comm., 2018. 9(1):2913.

[7] Dubrovinskaia, N, Dubrovinsky, L, Solopova, NA, et al., Terapascal static pressure generation with ultrahigh yield strength nanodiamond. Science Advances, 2016. 2(7):e1600341.

[8] Dewaele, A, Equations of state of simple solids (Including Pb, $\mathrm{NaCl}$ and LiF) compressed in helium or neon in the Mbar range. Minerals, 2019. 9(11):684.

[9] Occelli, F, Loubeyre, P, and LeToullec, R, Properties of diamond under hydrostatic pressures up to 140 GPa. Nat. Mat., 2003. 2(3):151-154. 
[10] Jacobsen, SD, Holl, CM, Adams, KA, et al., Compression of single-crystal magnesium oxide to 118 GPa and a ruby pressure gauge for helium pressure media. Am. Min., 2008. 93(11-12):18231828.

[11] Li, B, Woody, K, and Kung, J, Elasticity of MgO to $11 \mathrm{GPa}$ with an independent absolute pressure scale: Implications for pressure calibration. J. Geophys. Res., 2006. 111(B11).

[12] Zha, C-S, Mao, H-k, and Hemley, RJ, Elasticity of MgO and a primary pressure scale to $55 \mathrm{GPa}$. Proc. Natl. Acad. Sci., 2000. 97(25):13494-13499.

[13] Fat'yanov, OV and Asimow, PD, Equation of state of Mo from shock compression experiments on preheated samples. J. Appl. Phys., 2017. 121(11):115904.

[14] Mashimo, T, Liu, X, Kodama, M, et al., Effect of shear strength on Hugoniot-compression curve and the equation of state of tungsten (W). J. Appl. Phys., 2016. 119(3):035904.

[15] Duffy, TS and Smith, RF, Ultra-high pressure dynamic compression of geological materials. Frontiers in Earth Science, 2019. 7(23).

[16] Kraus, RG, Davis, JP, Seagle, CT, et al., Dynamic compression of copper to over 450 GPa: A highpressure standard. Phys. Rev. B, 2016. 93(13):134105.

[17] Kirsch, LE, Ali, SJ, Fratanduono, DE, et al., Refractive index of lithium fluoride to 900 gigapascal and implications for dynamic equation of state measurements. J. Appl. Phys., 2019. 125(17):175901.

[18] Smith, RF, Eggert, JH, Jeanloz, R, et al., Ramp compression of diamond to five terapascals. Natur, 2014. 511(7509):330-333.

[19] Kunc, K, Loa, I, and Syassen, K, Equation of state and phonon frequency calculations of diamond at high pressures. Phys. Rev. B, 2003. 68(9):094107.

[20] Decker, DL, Bassett, WA, Merrill, L, et al., High - pressure calibration: A critical review. J. Phys. Chem. Ref. Data, 1972. 1(3):773-836.

[21] Brown, JM, The NaCl pressure standard. J. Appl. Phys., 1999. 86(10):5801-5808.

[22] Marcondes, ML and Wentzcovitch, RM, Hybrid ab-initio/experimental high temperature equations of state: Application to the NaCl pressure scale. J. Appl. Phys., 2015. 117(21):215902.

[23] Forman, RA, Piermarini, GJ, Barnett, JD, et al., Pressure Measurement Made by the Utilization of Ruby Sharp-Line Luminescence. Science, 1972. 176(4032):284-285.

[24] Piermarini, GJ, Block, S, Barnett, JD, et al., Calibration of the pressure dependence of the R1 ruby fluorescence line to 195 kbar. J. Appl. Phys., 1975. 46(6):2774-2780.

[25] Mao, HK, Xu, J, and Bell, PM, Calibration of the ruby pressure gauge to 800 kbar under quasihydrostatic conditions. J. Geophys. Res., 1986. 91:4673-4676.

[26] Chai, $M$ and Brown, JM, Effects of static non-hydrostatic stress on the $R$ lines of ruby single crystals. Geophys. Res. Lett., 1996. 23(24):3539-3542.

[27] Takemura, K, Pressure scales and hydrostaticity. High Pressure Res., 2007. 27(4):465-472.

[28] Dewaele, A and Loubeyre, P, Pressurizing conditions in helium-pressure-transmitting medium. High Pressure Res., 2007. 27(4):419-429.

[29] Dewaele, A, Loubeyre, P, and Mezouar, M, Equations of state of six metals above 94 GPa. Phys. Rev. B, 2004. 70(9):094112.

[30] Dewaele, A, Torrent, M, Loubeyre, P, et al., Compression curves of transition metals in the Mbar range: Experiments and projector augmented-wave calculations. Phys. Rev. B, 2008.

78(10):104102.

[31] Dorogokupets, PI, P-V-T equations of state of $M g O$ and thermodynamics. Phys. Chem. Min., 2010. 37(9):677-684.

[32] Dorogokupets, $\mathrm{Pl}$ and Dewaele, A, Equations of state of $\mathrm{MgO}, \mathrm{Au}, \mathrm{Pt}, \mathrm{NaCl}-\mathrm{B1}$, and $\mathrm{NaCl}-\mathrm{B2}$ : Internally consistent high-temperature pressure scales. High Pressure Res., 2007. 27(4):431-446. 
[33] Holzapfel, WB, Refinement of the ruby luminescence pressure scale. J. Appl. Phys., 2003. 93(3):1813-1818.

[34] Holzapfel, WB, Equations of state for $\mathrm{Cu}, \mathrm{Ag}$, and $\mathrm{Au}$ and problems with shock wave reduced isotherms. High Pressure Res., 2010. 30(3):372-394.

[35] Kunc, K, Loa, I, and Syassen, K, Diamond under pressure: Ab-initio calculations of the equation of state and optical phonon frequency revisited. High Pressure Res., 2004. 24(1):101-110.

[36] Silvera, IF, Chijioke, AD, Nellis, WJ, et al., Calibration of the ruby pressure scale to $150 \mathrm{GPa}$. physica status solidi (b), 2007. 244(1):460-467.

[37] Syassen, K, Ruby under pressure. High Pressure Res., 2008. 28(2):75-126.

[38] Chijioke, AD, Nellis, WJ, and Silvera, IF, High-pressure equations of state of Al, Cu, Ta, and W. J. Appl. Phys., 2005. 98(7):073526.

[39] Holzapfel, WB, Progress in the realization of a practical pressure scale for the range 1-300 GPa. High Pressure Res., 2005. 25(2):87-99.

[40] Sokolova, TS, Dorogokupets, $\mathrm{PI}$, and Litasov, KD, Self-consistent pressure scales based on the equations of state for ruby, diamond, $\mathrm{MgO}, \mathrm{B2}-\mathrm{NaCl}$, as well as $\mathrm{Au}, \mathrm{Pt}$, and other metals to 4 Mbar and 3000 K. Russian Geology and Geophysics, 2013. 54(2):181-199.

[41] Mao, HK, Bell, PM, Shaner, JW, et al., Specific volume measurements of $\mathrm{Cu}, \mathrm{Mo}, \mathrm{Pd}$, and $\mathrm{Ag}$ and calibration of the ruby R1 fluorescence pressure gauge from 0.06 to 1 Mbar. J. Appl. Phys., 1978. 49(6):3276-3283.

[42] Fratanduono, DE, Smith, RF, Ali, SJ, et al., Probing the solid phase of noble metal copper at terapascal conditions. Phys. Rev. Lett., 2020. 124(1):015701.

[43] Al'tshuler, LV, Brusnikin, SE, and Kuz'menkov, EA, Isotherms and Grüneisen functions for 25 metals. JAMTP, 1987. 28(1):129-141.

[44] Chijioke, AD, Nellis, WJ, Soldatov, A, et al., The ruby pressure standard to 150GPa. J. Appl. Phys., 2005. 98(11):114905.

[45] Wang, Y, Ahuja, R, and Johansson, B, Reduction of shock-wave data with mean-field potential approach. J. Appl. Phys., 2002. 92(11):6616-6620.

[46] Carter, WJ, Marsh, SP, Fritz, JN, et al., The equation of state of selected materials for high pressure references. NBS Spec. Publ. , 1971. 36147.

[47] Hixson, RS and Fritz, JN, Shock compression of tungsten and molybdenum. J. Appl. Phys., 1992. 71(4):1721-1728.

[48] See the Supplemental Materials: Strength correction.

[49] Holzapfel, WB, Equations of state for solids under strong compression. High Pressure Res., 1998. 16(2):81-126.

[50] Bolef, DI and Klerk, JD, Elastic Constants of Single - Crystal Mo and W between $77^{\circ}$ and $500^{\circ}$ K. J. Appl. Phys., 1962. 33(7):2311-2314.

[51] Featherston, FH and Neighbours, JR, Elastic constants of tantalum, tungsten, and molybdenum. Phys. Rev., 1963. 130(4):1324-1333.

[52] Dickinson, JM and Armstrong, PE, Temperature Dependence of the Elastic Constants of Molybdenum. J. Appl. Phys., 1967. 38(2):602-606.

[53] Al'tshuler, LV, Brazhnik, MI, and Telegin, GS, Strength and elasticity of iron and copper at high shock-wave compression pressures. JAMTP, 1971. 12(6):921-926.

[54] Morris, CE and Fritz, JN, Relation of the "solid Hugoniot" to the "fluid Hugoniot" for aluminum and copper. J. Appl. Phys., 1980. 51(2):1244-1246.

[55] Furnish, MD, Chhabildas, LC, Steinberg, DJ, et al., Dynamic behavior of fully dense molybdenum, in Shock Compression of Condensed Matter-1991, S.C. Schmidt, et al., Editors. 1992, Elsevier: Amsterdam. p. 419-422. 
[56] Kleiser, GJ, Chhabildas, LC, Reinhart, WD, et al., Using time-resolved wave profile measurements to determine elusive phase transitions in molybdenum. Procedia Engineering, 2013. 58:617-623.

[57] Wang, Y, Chen, D, and Zhang, X, Calculated Equation of State of Al, Cu, Ta, Mo, and W to 1000 GPa. Phys. Rev. Lett., 2000. 84(15):3220-3223.

[58] Takemura, $K$ and Dewaele, A, Isothermal equation of state for gold with a He-pressure medium. Phys. Rev. B, 2008. 78(10):104119.

[59] Grimsditch, MH and Ramdas, AK, Brillouin scattering in diamond. Phys. Rev. B, 1975. 11(8):31393148.

[60] McSkimin, $\mathrm{HJ}$ and Jr., PA, Elastic moduli of diamond as a function of pressure and temperature. J. Appl. Phys., 1972. 43(7):2944-2948.

[61] Vogelgesang, R, Ramdas, AK, Rodriguez, S, et al., Brillouin and Raman scattering in natural and isotopically controlled diamond. Phys. Rev. B, 1996. 54(6):3989-3999.

[62] Tse, JS and Holzapfel, WB, Equation of state for diamond in wide ranges of pressure and temperature. J. Appl. Phys., 2008. 104(4):043525.

[63] Maezono, R, Ma, A, Towler, MD, et al., Equation of state and Raman frequency of diamond from quantum Monte Carlo simulations. Phys. Rev. Lett., 2007. 98(2):025701.

[64] Decker, DL, High - pressure equation of state for $\mathrm{NaCl}, \mathrm{KCl}$, and $\mathrm{CsCl}$. J. Appl. Phys., 1971. 42(8):3239-3244.

[65] Dorogokupets, $\mathrm{PI}$ and Oganov, AR, Ruby, metals, and $\mathrm{MgO}$ as alternative pressure scales: $A$ semiempirical description of shock-wave, ultrasonic, $x$-ray, and thermochemical data at high temperatures and pressures. Phys. Rev. B, 2007. 75(2):024115.

[66] Grasset, O, Calibration of the $R$ ruby fluorescence lines in the pressure range [0-1 GPa] and the temperature range [250-300 K]. High Pressure Res., 2001. 21(3-4):139-157.

[67] Funamori, $\mathrm{N}$ and Jeanloz, $\mathrm{R}$, High-pressure transformation of $\mathrm{Al}_{2} \mathrm{O}_{3}$. Science, 1997. 278(5340):1109-1111.

[68] Ono, S, Oganov, AR, Koyama, T, et al., Stability and compressibility of the high-pressure phases of Al2O3 up to $200 \mathrm{GPa}$ : Implications for the electrical conductivity of the base of the lower mantle. Earth Planet. Sci. Lett., 2006. 246(3):326-335.

[69] Sherman, WF, The diamond Raman band as a high-pressure calibrant. J. Phys. C, 1985. 18(30):L973-L978.

[70] Akahama, $\mathrm{Y}$ and Kawamura, H, Diamond anvil Raman gauge in multimegabar pressure range. High Pressure Res., 2007. 27(4):473-482.

[71] Goncharov, AF, Crowhurst, JC, Dewhurst, JK, et al., Raman spectroscopy of cubic boron nitride under extreme conditions of high pressure and temperature. Phys. Rev. B, 2005. 72(10):100104.

[72] Datchi, F and Canny, B, Raman spectrum of cubic boron nitride at high pressure and temperature. Phys. Rev. B, 2004. 69(14):144106.

[73] Shen, XA and Gupta, YM, Effect of crystal orientation on ruby R-line shifts under shock compression and tension. Phys. Rev. B, 1993. 48(5):2929-2940.

[74] Chervin, JC, Canny, B, and Mancinelli, M, Ruby-spheres as pressure gauge for optically transparent high pressure cells. High Pressure Res., 2001. 21(6):305-314.

[75] Cook, RF and Michaels, CA, Review: coefficients for stress, temperature, and composition effects in fluorescence measurements of alumina. J. Res. Nat. Inst. Stand. Tech., 2017. 122(43). 


\section{Supplemental Materials}

\section{Toward an international practical pressure scale: A proposal for an IPPS ruby gauge (IPPS-Ruby2020) \\ AIRAPT IPPS Task Group}

Contents of the file:

Figure S1. Estimated errors from thermal correction in the reduced-shockwave data $\quad$............................ 2

Figure S2. The room-temperature isotherms of Mo reduced from Hugoniot data ..................................... 3

Figure S3. The room-temperature isotherms of $\mathrm{Cu}$ reduced from ramp compression data ...................... 4

Table S1. Numerical tabulation of the room-temperature isotherms of Mo …......................................... 5

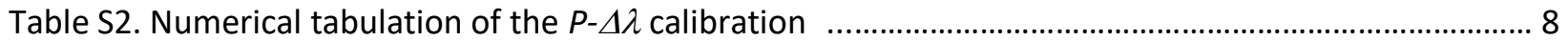

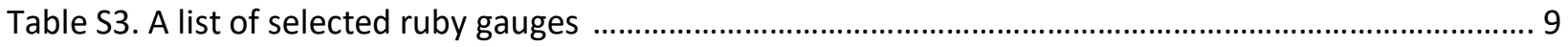

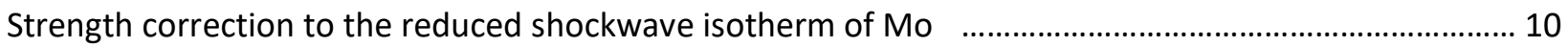




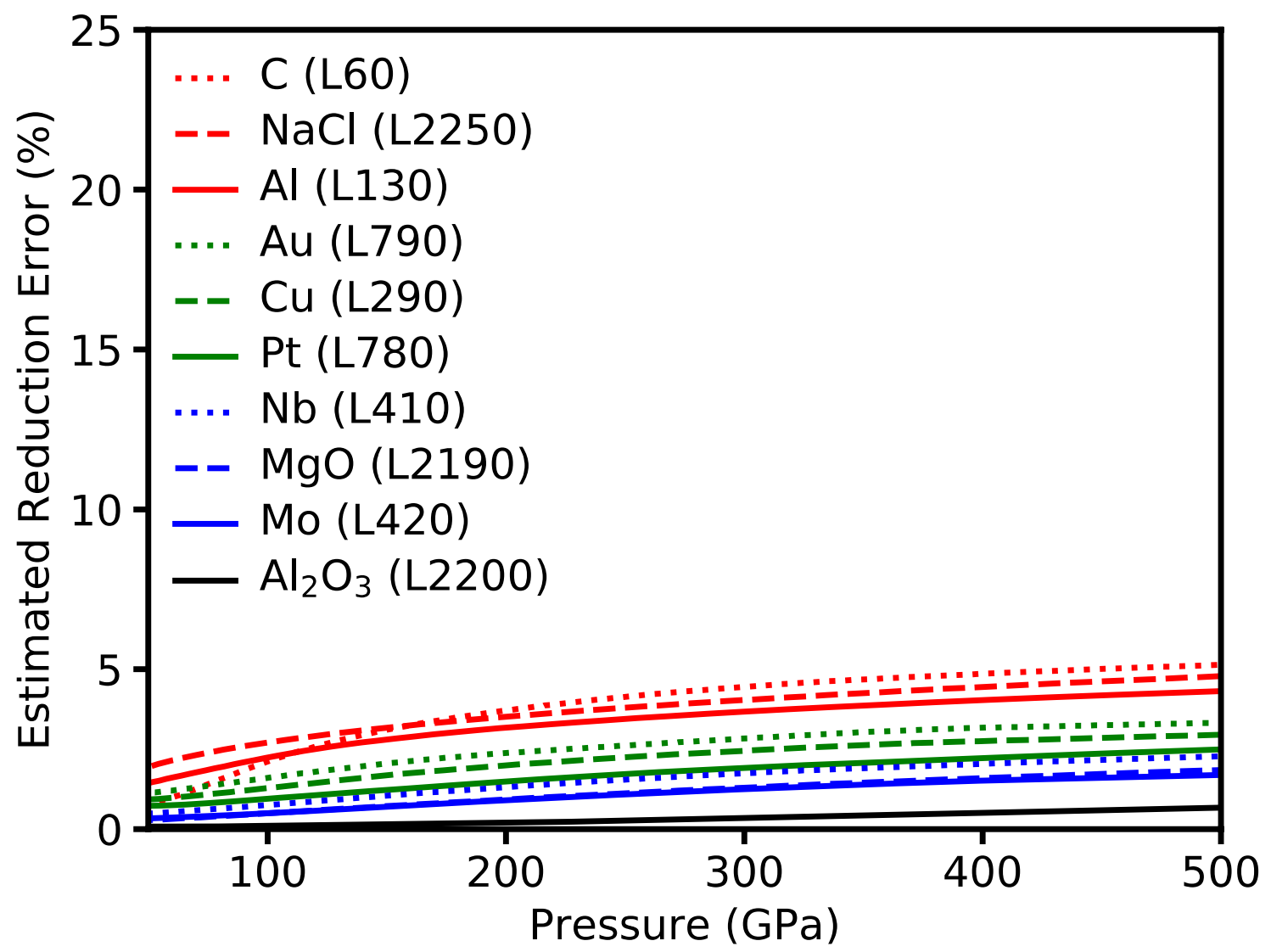

Figure S1. Estimated errors from thermal correction in the reduced-shockwave data of various materials [1]. These estimates are based on $\sigma=\frac{P_{i e}}{P_{t}} \phi$, where $P_{i e}$ is the total thermal pressure contributed from both ions and electrons, $P_{t}$ is the total pressure, and $\phi$ is the estimated thermal uncertainty factor (expressed as percentage error in thermal properties). $P_{i e}$ and $P_{t}$ are computed from existing EOSs in the Livermore Equation Of State (LEOS) library produced by Lawrence Livermore National Laboratory. An uncertainty of $10 \%$ on thermal properties is used as estimate for $\phi$, which gives correction errors of $\pm 0.5 \%$ and $\pm 1.28 \%$ for $\mathrm{Mo}$ and $\mathrm{Cu}$ at $100 \mathrm{GPa}$, respectively, in agreement with the reported values in an earlier study [2]. The uncertainties from thermal correction at $150 \mathrm{GPa}$ for $\mathrm{Mo}$ and $\mathrm{Cu}$ are $\pm 0.7 \%$ and $\pm 1.7 \%$, respectively. 


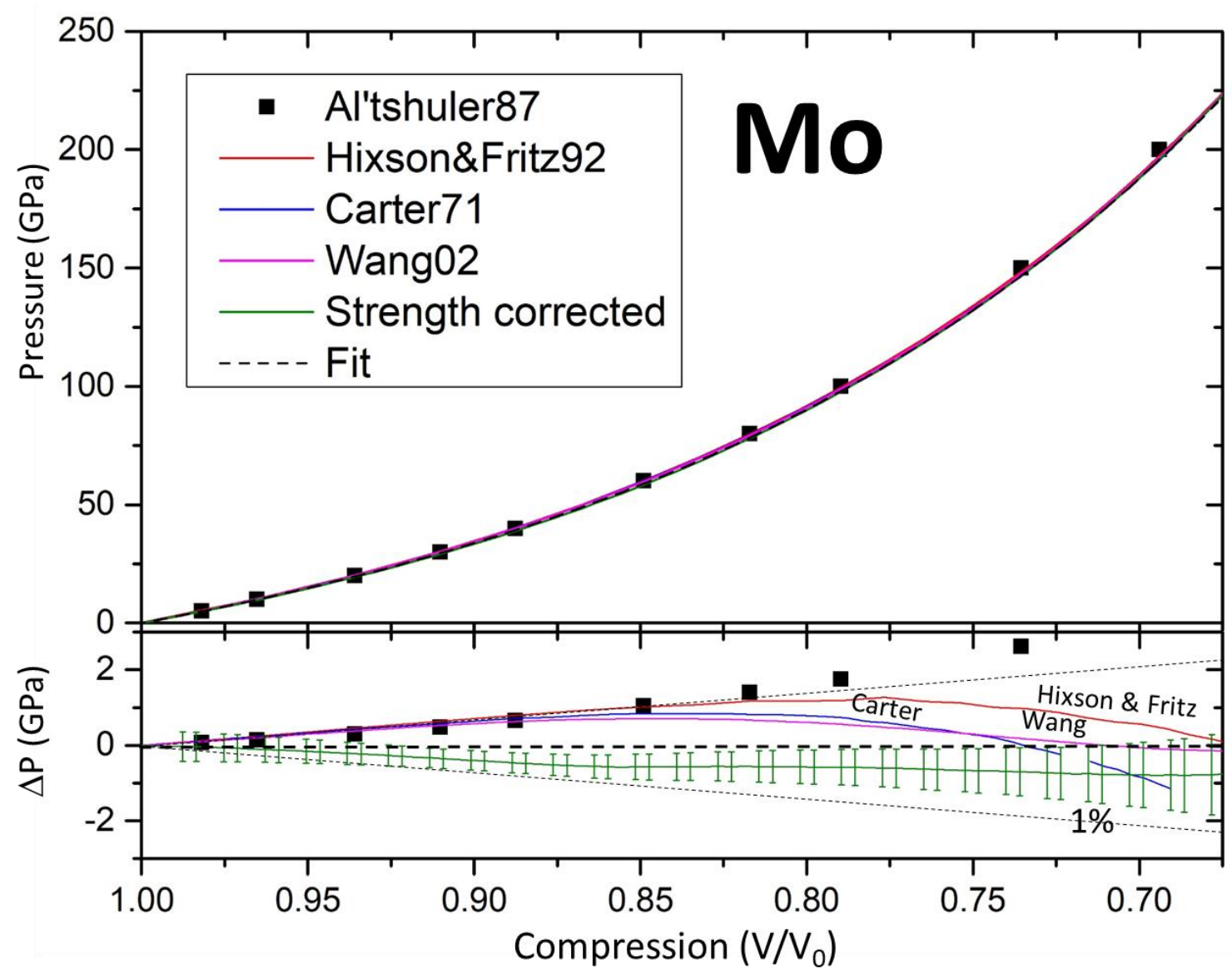

Figure S2. Room-temperature isotherms of Mo reduced from Hugoniot data [3-6]. The reduced isotherms are in mutual agreement within $\pm 1 \%$ up to $250 \mathrm{GPa}$, except Al'tshuler et al's data [6] which show slightly stiffer at pressures above $150 \mathrm{GPa}$. A correction for material strength was applied in the reduced isotherm from the recent reported Hugoniot data [7] (green line with error bars shown at the bottom). See the section of "strength correction" in this Supplement for more details. The fit to the combined isotherms using Holzapfel's EOS-AP2 gives $K_{T O}$ ' of $4.00(1)$, with the $K_{T O}$ value of $260 \mathrm{GPa}$ constrained by experimental data at standard conditions. Pressure differences relative to the fit are shown at the bottom. 


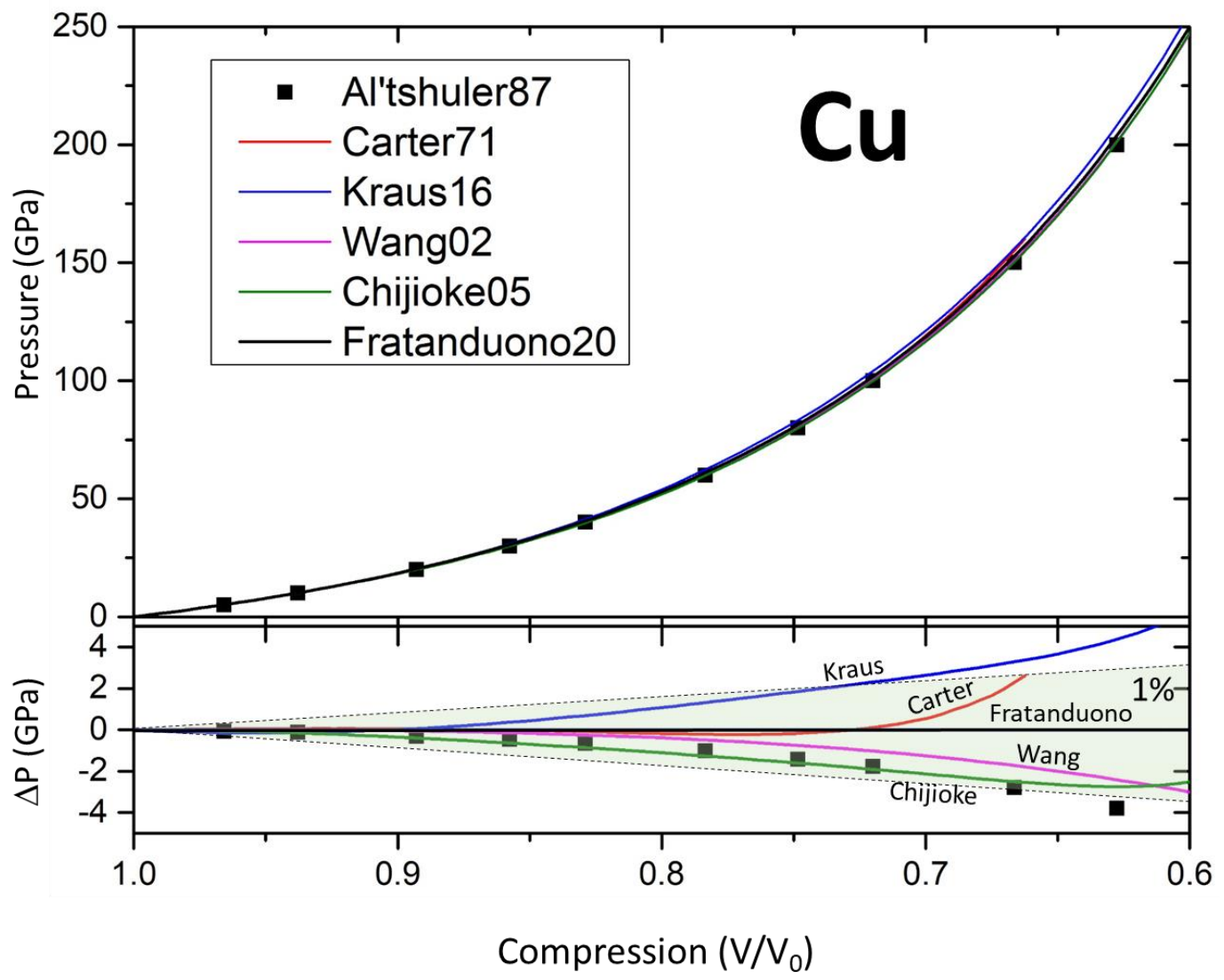

Figure S3. Room-temperature isotherms of $\mathrm{Cu}$ reduced from ramp compression data $[8,9]$ compared with those reduced from Hugoniot data [3, 5, 6, 10]. A recent study by Fratanduono et al [8] is consistent with the reduced Hugoniot data within $\pm 1 \%$ up to $150 \mathrm{GPa}$. Pressure differences relative to the data from [8] are shown at the bottom. 
Table S1. Numerical tabulation of the room-temperature isotherms of Mo reduced from dynamic compression data

\begin{tabular}{|c|c|c|c|c|c|c|}
\hline \multicolumn{2}{|c|}{ Hixson and Fritz [4] } & \multicolumn{2}{|c|}{ Carter et al [3] } & \multicolumn{3}{|c|}{$\begin{array}{l}\text { Reduced from Fat'yanov and Asimow } \\
\text { [7], see "strength correction" below for } \\
\text { more details }\end{array}$} \\
\hline $\mathrm{P}(\mathrm{GPa})$ & $\begin{array}{c}\rho_{0} / \rho\left(\rho_{0}=10215\right. \\
\left.\mathrm{kg} / \mathrm{m}^{3}\right)\end{array}$ & $P(G P a)$ & $\begin{array}{c}\rho_{0} / \rho\left(\rho_{0}=10206\right. \\
\left.\mathrm{kg} / \mathrm{m}^{3}\right)\end{array}$ & $\mathrm{P}(\mathrm{GPa})$ & $\begin{array}{c}\rho_{0} / \rho\left(\rho_{0}=\right. \\
\left.10205 \mathrm{~kg} / \mathrm{m}^{3}\right)\end{array}$ & $\begin{array}{l}\text { P uncertainty } \\
\text { 1-sigma (GPa) }\end{array}$ \\
\hline 0 & 1.00000 & 0 & 1.00000 & 0.0 & 1.00000 & 0.00 \\
\hline 10.0 & 0.96559 & 0.5 & 0.99814 & 1.1 & 0.99588 & 0.22 \\
\hline 20.0 & 0.93621 & 1.0 & 0.99629 & 2.2 & 0.99175 & 0.39 \\
\hline 30.0 & 0.91059 & 1.5 & 0.99445 & 3.3 & 0.98763 & 0.39 \\
\hline 40.0 & 0.88787 & 2.0 & 0.99261 & 4.4 & 0.98350 & 0.39 \\
\hline 50.0 & 0.86752 & 2.5 & 0.99078 & 5.6 & 0.97938 & 0.38 \\
\hline 60.0 & 0.84906 & 3.0 & 0.98905 & 6.8 & 0.97525 & 0.37 \\
\hline 70.0 & 0.83218 & 3.5 & 0.98723 & 8.0 & 0.97113 & 0.36 \\
\hline 80.0 & 0.81674 & 4.0 & 0.98552 & 9.2 & 0.96700 & 0.35 \\
\hline 90.0 & 0.80237 & 4.5 & 0.98381 & 10.5 & 0.96288 & 0.34 \\
\hline 100.0 & 0.78906 & 5.0 & 0.98201 & 11.8 & 0.95875 & 0.34 \\
\hline 110.0 & 0.77675 & 5.5 & 0.98031 & 13.1 & 0.95463 & 0.33 \\
\hline 120.0 & 0.76500 & 6.0 & 0.97862 & 14.5 & 0.95050 & 0.32 \\
\hline 130.0 & 0.75405 & 6.5 & 0.97693 & 15.8 & 0.94638 & 0.31 \\
\hline 140.0 & 0.74367 & 7.0 & 0.97519 & 17.2 & 0.94225 & 0.30 \\
\hline 150.0 & 0.73394 & 7.5 & 0.97367 & 18.7 & 0.93813 & 0.29 \\
\hline 160.0 & 0.72467 & 8.0 & 0.97200 & 20.1 & 0.93400 & 0.29 \\
\hline 170.0 & 0.71584 & 8.5 & 0.97034 & 21.6 & 0.92988 & 0.28 \\
\hline 180.0 & 0.70746 & 9.0 & 0.96877 & 23.1 & 0.92575 & 0.27 \\
\hline 190.0 & 0.69951 & 9.5 & 0.96712 & 24.7 & 0.92163 & 0.27 \\
\hline 200.0 & 0.69189 & 10.0 & 0.96556 & 26.3 & 0.91750 & 0.26 \\
\hline 210.0 & 0.68456 & 10.5 & 0.96401 & 27.9 & 0.91338 & 0.26 \\
\hline 220.0 & 0.67757 & 11.0 & 0.96238 & 29.6 & 0.90925 & 0.26 \\
\hline 230.0 & 0.67085 & 11.5 & 0.96084 & 31.3 & 0.90513 & 0.26 \\
\hline 240.0 & 0.66443 & 12.0 & 0.95930 & 33.0 & 0.90100 & 0.26 \\
\hline 250.0 & 0.65823 & 12.5 & 0.95777 & 34.8 & 0.89688 & 0.26 \\
\hline 260.0 & 0.65226 & 13.0 & 0.95624 & 36.6 & 0.89275 & 0.26 \\
\hline 270.0 & 0.64652 & 13.5 & 0.95472 & 38.4 & 0.88863 & 0.27 \\
\hline 280.0 & 0.64096 & 14.0 & 0.95330 & 40.3 & 0.88450 & 0.27 \\
\hline 290.0 & 0.63566 & 14.5 & 0.95179 & 42.2 & 0.88038 & 0.28 \\
\hline 300.0 & 0.63052 & 15.0 & 0.95028 & 44.2 & 0.87625 & 0.28 \\
\hline & & 20.0 & 0.93616 & 46.2 & 0.87213 & 0.29 \\
\hline & & 25.0 & 0.92287 & 48.3 & 0.86800 & 0.30 \\
\hline & & 30.0 & 0.91044 & 50.4 & 0.86387 & 0.31 \\
\hline & & 35.0 & 0.89873 & 52.6 & 0.85975 & 0.32 \\
\hline & & 40.0 & 0.88771 & 54.8 & 0.85562 & 0.33 \\
\hline & & 45.0 & 0.87718 & 57.0 & 0.85150 & 0.34 \\
\hline & & 50.0 & 0.86727 & 59.4 & 0.84737 & 0.34 \\
\hline & & 55.0 & 0.85779 & 61.7 & 0.84325 & 0.35 \\
\hline & & 60.0 & 0.84873 & 64.2 & 0.83912 & 0.36 \\
\hline & & 65.0 & 0.84007 & 66.6 & 0.83500 & 0.37 \\
\hline & & 70.0 & 0.83178 & 69.2 & 0.83087 & 0.38 \\
\hline & & 75.0 & 0.82386 & 71.8 & 0.82675 & 0.39 \\
\hline & & 80.0 & 0.81622 & 74.4 & 0.82262 & 0.40 \\
\hline & & 85.0 & 0.80891 & 77.1 & 0.81850 & 0.40 \\
\hline & & 90.0 & 0.80185 & 79.9 & 0.81437 & 0.41 \\
\hline & & 95.0 & 0.79505 & 82.7 & 0.81025 & 0.42 \\
\hline & & 100.0 & 0.78847 & 85.6 & 0.80612 & 0.43 \\
\hline & & 105.0 & 0.78207 & 88.5 & 0.80200 & 0.44 \\
\hline & & 110.0 & 0.77594 & 91.6 & 0.79787 & 0.45 \\
\hline & & 115.0 & 0.76997 & 94.6 & 0.79375 & 0.46 \\
\hline & & 120.0 & 0.76421 & 97.8 & 0.78962 & 0.47 \\
\hline
\end{tabular}




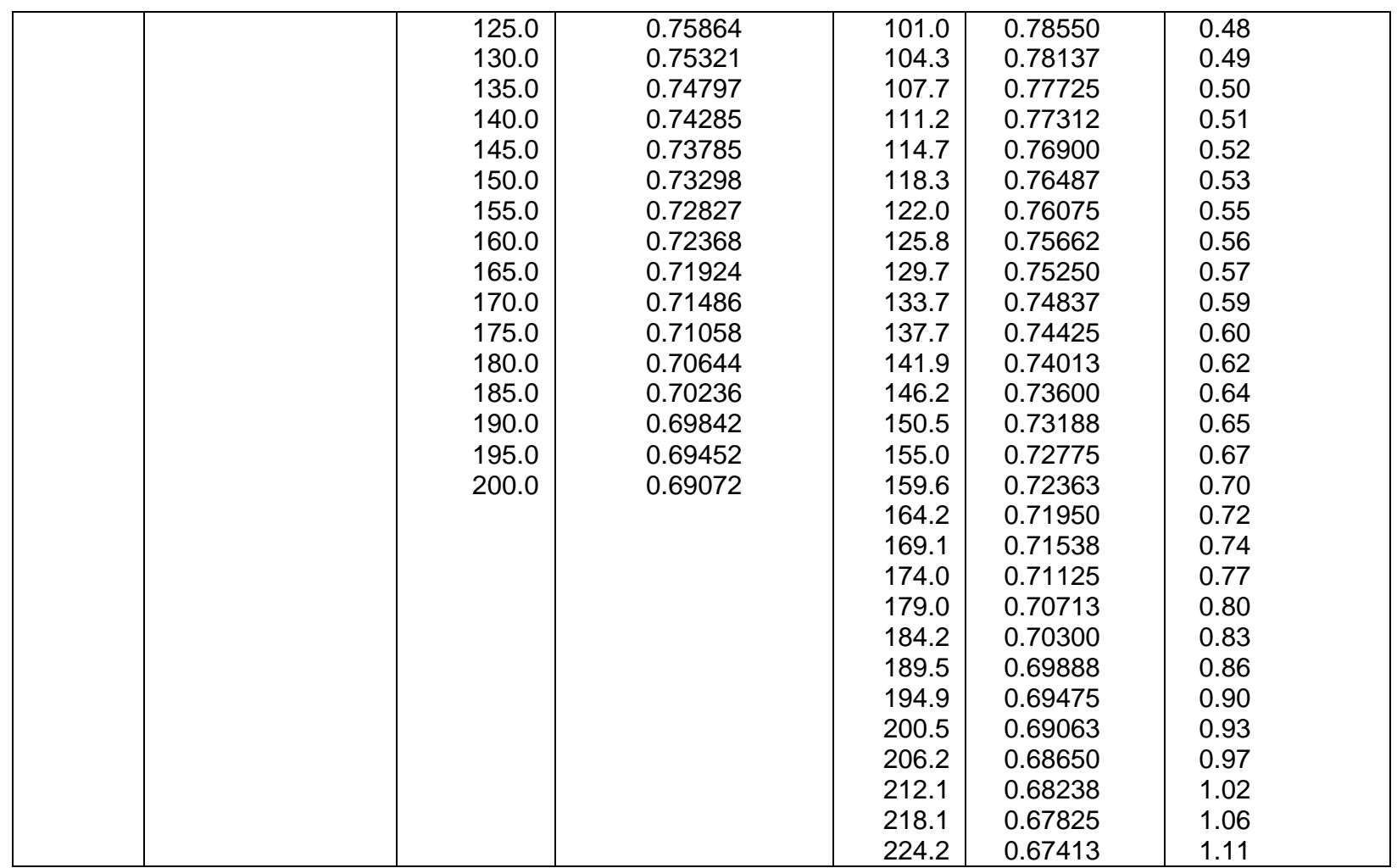


Table S1 - continued. Numerical tabulation of the room-temperature isotherms of Mo reduced from dynamic compression data

\begin{tabular}{|r|c|c|}
\hline \multicolumn{2}{|c|}{ Al'tshuler et al [6] } & Wang et al [5] \\
\hline $\mathrm{P}(\mathrm{GPa})$ & $\begin{array}{c}\rho_{0} / \rho\left(\rho_{0}=\right. \\
\left.10206 \mathrm{~kg} / \mathrm{m}^{3}\right)\end{array}$ & $\begin{array}{c}\text { Universal EOS } \\
4^{\text {th }} \text { order }\end{array}$ \\
\hline 5 & 0.9819 & $K_{T O}: 264.87 \mathrm{GPa}$ \\
10 & 0.9653 & \\
20 & 0.9358 & $\eta: 4.7127$ \\
30 & 0.9102 & \\
40 & 0.8876 & $\beta:-8.1794$ \\
60 & 0.8491 & \\
80 & 0.8171 & $\psi: 83.532$ \\
100 & 0.7898 & \\
150 & 0.7355 & $\delta:-189.67$ \\
200 & 0.6940 & \\
300 & 0.6328 & \\
400 & 0.5885 & \\
\hline
\end{tabular}

a: The universal EOS form: $P(x)=3 K_{T O}\left[\left(1-x^{1 / 3}\right) / x^{2 / 3}\right] \exp \left[\eta\left(1-x^{1 / 3}\right)+\beta\left(1-x^{1 / 3}\right)^{2}+\psi\left(1-x^{1 / 3}\right)^{3}+\delta(1-\right.$ $\left.\left.x^{1 / 3}\right)^{4}\right]$, where $x=\rho_{0} / \rho$. 
Table S2. Numerical tabulation of the $P-\Delta \lambda$ calibration. Sources of the $V-\Delta \lambda$ relations are given, with $\lambda_{0}=$ $694.25 \mathrm{~nm}$. See the main text for the $P-V$ isotherms at room temperature.

\begin{tabular}{|c|c|c|c|c|c|c|c|c|c|c|}
\hline \multicolumn{3}{|c|}{ Mo } & \multicolumn{3}{|c|}{$\mathrm{Cu}$} & \multicolumn{2}{|c|}{$\mathbf{M g O}^{c}$} & \multicolumn{3}{|c|}{ Diamond $^{d}$} \\
\hline $\begin{array}{l}V / V_{o} \\
{[11]}\end{array}$ & $\begin{array}{l}\lambda-\lambda_{0} \\
(\mathrm{~nm}) \\
{[11]}\end{array}$ & $\begin{array}{c}P(G P a)^{a} \\
K_{0}=260 \\
G P a \\
K_{0}^{\prime}=4.00\end{array}$ & $\begin{array}{l}V / V_{0} \\
{[12]}\end{array}$ & $\begin{array}{l}\lambda-\lambda_{0} \\
(n m) \\
{[12]}\end{array}$ & $P(G P a)^{b}$ & $\begin{array}{l}\lambda-\lambda_{0} \\
(\mathrm{~nm}) \\
{[13]}\end{array}$ & $\begin{array}{c}P \\
(G P a) \\
{[13]}\end{array}$ & $\begin{array}{l}V / V_{0} \\
{[14]}\end{array}$ & $\begin{array}{l}\lambda-\lambda_{0} \\
(n m) \\
{[14]}\end{array}$ & $\begin{array}{c}P(G P a)^{a} \\
K_{0}=443.3 \\
G P a \\
K_{0}^{\prime}=3.7\end{array}$ \\
\hline 0.99333 & 0.65 & 1.76 & 0.98874 & 0.56 & 1.56 & 0.72 & 2.00 & 1.00000 & 0.00 & 0.00 \\
\hline 0.98187 & 1.88 & 4.93 & 0.97824 & 1.09 & 3.11 & 1.17 & 3.22 & 0.99898 & 0.12 & 0.46 \\
\hline 0.97270 & 2.88 & 7.61 & 0.97036 & 1.54 & 4.35 & 2.03 & 5.50 & 0.99742 & 0.37 & 1.15 \\
\hline 0.97062 & 3.09 & 8.23 & 0.95935 & 2.17 & 6.17 & 2.68 & 7.47 & 0.99584 & 0.61 & 1.86 \\
\hline 0.96646 & 3.58 & 9.50 & 0.94817 & 2.89 & 8.16 & 2.87 & 7.89 & 0.99429 & 0.86 & 2.56 \\
\hline 0.95908 & 4.36 & 11.81 & 0.93733 & 3.67 & 10.22 & 2.94 & 8.03 & 0.99274 & 1.10 & 3.27 \\
\hline 0.95207 & 5.16 & 14.09 & 0.92556 & 4.47 & 12.63 & 3.46 & 9.37 & 0.99119 & 1.34 & 3.99 \\
\hline 0.94364 & 6.19 & 16.94 & 0.91650 & 5.12 & 14.60 & 4.05 & 11.15 & 0.98911 & 1.71 & 4.95 \\
\hline 0.93738 & 6.95 & 19.13 & 0.90481 & 6.11 & 17.31 & 5.69 & 15.97 & 0.98651 & 2.19 & 6.17 \\
\hline 0.93346 & 7.46 & 20.54 & 0.89516 & 6.95 & 19.70 & 5.75 & 16.11 & 0.98288 & 2.79 & 7.90 \\
\hline 0.92656 & 8.34 & 23.10 & 0.88559 & 7.72 & 22.22 & 6.47 & 18.21 & 0.98080 & 3.15 & 8.91 \\
\hline 0.91825 & 9.38 & 26.29 & 0.87669 & 8.61 & 24.70 & 7.19 & 20.18 & 0.97665 & 3.75 & 10.94 \\
\hline 0.91151 & 10.40 & 28.99 & 0.86831 & 9.36 & 27.16 & 7.19 & 20.46 & 0.97249 & 4.69 & 13.02 \\
\hline 0.89876 & 12.20 & 34.35 & 0.85925 & 10.37 & 29.97 & 7.91 & 22.28 & 0.95850 & 7.13 & 20.32 \\
\hline 0.90363 & 11.42 & 32.26 & 0.85307 & 10.97 & 31.98 & 7.91 & 22.56 & 0.94085 & 10.30 & 30.24 \\
\hline 1.00000 & 0.00 & 0.00 & 0.84494 & 11.96 & 34.75 & 8.62 & 24.52 & 0.92839 & 12.83 & 37.78 \\
\hline 0.89667 & 12.20 & 35.26 & 1.00000 & 0.00 & 0.00 & 8.69 & 24.80 & 0.90140 & 17.94 & 55.71 \\
\hline 0.87187 & 15.59 & 46.88 & 0.90244 & 6.31 & 17.88 & 9.02 & 25.64 & 0.88742 & 21.01 & 65.97 \\
\hline 0.85603 & 18.14 & 55.13 & 0.85654 & 10.62 & 30.85 & 9.02 & 25.92 & 0.87287 & 24.09 & 77.40 \\
\hline 0.84086 & 20.60 & 63.69 & 0.83918 & 12.52 & 36.80 & 9.67 & 27.46 & 0.85938 & 26.89 & 88.77 \\
\hline 0.83039 & 22.29 & 70.02 & 0.82393 & 14.29 & 42.60 & 9.67 & 27.88 & 0.84744 & 29.53 & 99.47 \\
\hline 0.82109 & 23.79 & 75.95 & 0.81072 & 15.90 & 48.10 & 10.91 & 31.10 & 0.83863 & 31.65 & 107.79 \\
\hline 0.81323 & 25.29 & 81.20 & 0.79963 & 17.36 & 53.09 & 10.98 & 31.38 & 0.82774 & 34.36 & 118.59 \\
\hline 0.80428 & 26.98 & 87.46 & 0.78701 & 19.12 & 59.22 & 10.91 & 31.66 & 0.81683 & 36.92 & 130.03 \\
\hline 0.79761 & 28.24 & 92.32 & 0.77896 & 20.41 & 63.40 & 10.98 & 31.94 & 0.80697 & 39.59 & 140.92 \\
\hline 0.79016 & 29.69 & 97.96 & 0.76863 & 21.74 & 69.09 & 12.28 & 35.31 & 0.79763 & 41.86 & 151.75 \\
\hline 0.78034 & 31.33 & 105.77 & 0.76194 & 22.74 & 72.99 & 12.28 & 35.87 & & & \\
\hline 0.77109 & 33.48 & 113.52 & 0.75483 & 23.90 & 77.32 & 13.20 & 38.11 & & & \\
\hline 0.76029 & 35.59 & 123.08 & 0.74687 & 25.33 & 82.42 & 13.33 & 38.53 & & & \\
\hline & & & 0.73721 & 27.01 & 88.98 & 13.20 & 38.81 & & & \\
\hline & & & 0.72857 & 28.58 & 95.22 & 13.27 & 39.09 & & & \\
\hline & & & 0.72400 & 29.58 & 98.67 & 13.85 & 40.21 & & & \\
\hline & & & 0.71468 & 31.48 & 106.04 & 13.86 & 40.77 & & & \\
\hline & & & 0.70672 & 32.97 & 112.72 & 15.36 & 44.69 & & & \\
\hline & & & 0.70037 & 34.19 & 118.31 & 15.29 & 45.39 & & & \\
\hline & & & 0.69419 & 35.63 & 123.99 & 15.68 & 45.67 & & & \\
\hline & & & 0.68750 & 37.05 & 130.42 & 15.62 & 46.52 & & & \\
\hline & & & 0.68123 & 38.21 & 136.73 & 16.99 & 49.88 & & & \\
\hline & & & 0.67615 & 39.81 & 142.04 & 16.93 & 50.72 & & & \\
\hline & & & 0.67141 & 40.94 & 147.17 & 17.97 & 53.10 & & & \\
\hline & & & 0.66777 & 41.83 & 151.23 & 17.97 & 53.94 & & & \\
\hline & & & 0.66421 & 42.72 & 155.30 & 18.30 & 54.22 & & & \\
\hline & & & & & & 18.30 & 55.06 & & & \\
\hline
\end{tabular}

${ }^{a}$ Holzapfel's AP2 EOS (Eq. 1)

${ }^{b}$ The third order Vinet EOS (Table 1 in [8])

${ }^{c}$ Digitized from Fig. 6 in [13]

${ }^{d}$ Digitized from Fig. 2 in [14] 
Table S3. A list of selected ruby gauges. Some entries have no error assessment reported by the original authors

\begin{tabular}{|c|c|c|c|}
\hline Authors & Parameters & Equations & Comments \\
\hline $\begin{array}{c}\text { Mao et al } 1986 \\
{[15]}\end{array}$ & $\begin{array}{l}\text { A: } 1904 \mathrm{GPa} \\
\text { B: } 7.665\end{array}$ & $A / B\left[\left(\lambda / \lambda_{0}\right)^{B}-1\right]$ & $\mathrm{Cu}, \mathrm{Ag}$ in $\mathrm{Ar}$ to $80 \mathrm{GPa}$ \\
\hline $\begin{array}{c}\text { Aleksandrov et al } \\
1987[16]\end{array}$ & $\begin{array}{l}\text { A: } 1892(25) \mathrm{GPa} \\
B: 6.4(5)\end{array}$ & $A\left(\Delta \lambda / \lambda_{0}\right)\left[1+B\left(\Delta \lambda / \lambda_{0}\right)\right]$ & Diamond in $\mathrm{He}$ up to $42 \mathrm{GPa}$ \\
\hline $\begin{array}{c}\text { Kunc et al } 2004 \\
{[17]}\end{array}$ & $\begin{array}{l}A: 1860 \mathrm{GPa} \\
B: 7.7\end{array}$ & $A\left(\Delta v / v_{0}\right)\left[1+B\left(\Delta v / v_{0}\right)\right]$ & Diamond phonon, DFT \\
\hline $\begin{array}{c}\text { Holzapfel } 2005 \\
{[18]}\end{array}$ & $\begin{array}{l}\text { A: } 1845(25) \mathrm{GPa} \\
B: 14.7 \\
C: 7.5\end{array}$ & $\begin{array}{l}A /(B+C)[\exp (\eta)-1] \\
\eta=(B+C) / C\left[1-(\lambda o / \lambda)^{C}\right]\end{array}$ & $\begin{array}{l}\text { Analysis of published data, } \\
\text { including data from shockwave }\end{array}$ \\
\hline $\begin{array}{c}\text { Chijioke et al } \\
2005 \text { [19] }\end{array}$ & $\begin{array}{l}\text { A: } 1876(6.7) \mathrm{GPa} \\
\text { B: } 10.71(14)\end{array}$ & $A / B\left[\left(\lambda / \lambda_{0}\right)^{B}-1\right]$ & Revised shockwave data \\
\hline $\begin{array}{c}\text { Dorogokupets } \\
\text { and Oganov } 2007 \\
{[20]}\end{array}$ & $\begin{array}{l}\text { A: } 1884 \mathrm{GPa} \\
B: 5.5\end{array}$ & $A\left(\Delta \lambda / \lambda_{0}\right)\left[1+B\left(\Delta \lambda / \lambda_{0}\right)\right]$ & $\begin{array}{l}\text { Analysis of published data, self- } \\
\text { consistent approach }\end{array}$ \\
\hline $\begin{array}{l}\text { Dewaele et al } \\
2008[11]\end{array}$ & $\begin{array}{l}\text { A: } 1920 \mathrm{GPa} \\
\text { B: } 9.61\end{array}$ & $\begin{array}{l}P(G P a)= \\
A / B\left[\left(\lambda / \lambda_{0}\right)^{B}-1\right]\end{array}$ & $\begin{array}{l}P \text { - } \lambda \text { data from } 10 \text { metals in } \mathrm{He} \\
\text { medium, shockwave } \mathrm{P}-\mathrm{V} \text { data }\end{array}$ \\
\hline $\begin{array}{c}\text { Jacobsen et al } \\
2008[21] \\
\end{array}$ & $\begin{array}{l}\text { A: } 1904 \mathrm{GPa} \\
B: 10.32(7)\end{array}$ & $A / B\left[\left(\lambda / \lambda_{0}\right)^{B}-1\right]$ & $\mathrm{MgO}$ to $118 \mathrm{GPa}$, He medium \\
\hline $\begin{array}{c}\text { Syassen } 2008 \\
{[22]}\end{array}$ & $\begin{array}{l}A: 1870(30) \mathrm{GPa} \\
B: 5.9\end{array}$ & $A\left(\Delta \lambda / \lambda_{0}\right)\left[1+B\left(\Delta \lambda / \lambda_{0}\right)\right]$ & Averaged scale, published data \\
\hline $\begin{array}{c}\text { Holzapfel } 2010 \\
\text { [23] }\end{array}$ & $\begin{array}{l}A: 1836 \mathrm{GPa} \\
B: 17.1 \\
C: 11\end{array}$ & $\begin{array}{l}A /(B+C)[\exp (\eta)-1] \\
\eta=(B+C) / C\left[1-(\lambda d / \lambda)^{C}\right]\end{array}$ & $\begin{array}{l}\text { Analysis of published data, } \\
\text { excluding data from shockwave }\end{array}$ \\
\hline $\begin{array}{l}\text { Sokolova et al } \\
2013[24]\end{array}$ & $\begin{array}{l}\text { A: } 1870 \mathrm{GPa} \\
\text { B: } 6.0\end{array}$ & $A\left(\Delta \lambda / \lambda_{0}\right)\left[1+B\left(\Delta \lambda / \lambda_{0}\right)\right]$ & $\begin{array}{l}\text { Analysis of published data, self- } \\
\text { consistent approach }\end{array}$ \\
\hline $\begin{array}{c}\text { Kraus et al } 2016 \\
{[9]}\end{array}$ & $\begin{array}{l}A: 1915.1 \mathrm{GPa} \\
B: 10.603\end{array}$ & $A / B\left[\left(\lambda / \lambda_{0}\right)^{B}-1\right]$ & $\begin{array}{l}P-V \text { data of copper reduced from } \\
\text { ramp compression }\end{array}$ \\
\hline IPPS Ruby2020 & $\begin{array}{l}A: 1.87(1) \times 10^{3} \mathrm{GPa} \\
B: 5.63(3)\end{array}$ & $A\left(\Delta \lambda / \lambda_{0}\right)\left[1+B\left(\Delta \lambda / \lambda_{0}\right)\right]$ & $\begin{array}{l}P-\lambda \text { data from two metals (Mo } \\
\text { and } \mathrm{Cu} \text { ), } \mathrm{MgO} \text {, and diamond }\end{array}$ \\
\hline
\end{tabular}




\section{Strength correction to the reduced shockwave isotherm of Mo}

Using recently reported shock compression data [7], we have computed a reduced roomtemperature isotherm for Mo by accounting for the residual material strength. The strength correction to the pressure on the isotherm is about $-2.3 \%$ at $58 \mathrm{GPa}\left(V / V_{0} \approx 0.85\right)$ and about $-1.5 \%$ at $150 \mathrm{GPa}$ $\left(V / V_{0} \approx 0.73\right)$. In addition, we have employed a combined Debye and electronic specific heat model in the thermal correction analysis. Comparing our thermal correction data with those obtained using a simple Debye model by Hixson and Fritz [4], the difference in pressure on the isotherm is small: only $0.2 \mathrm{GPa}$ (or $1 / 3$ of its total uncertainty) at $150 \mathrm{GPa}$ and $0.6 \mathrm{GPa}$ (or $1 / 2$ of its total uncertainty) at $230 \mathrm{GPa}$.

Table S4 lists the parameters used in the analysis. We first compute the longitudinal stress $\sigma_{x}$ along the Hugoniot using the linear principal $U_{S}-U_{P}$ relation and the initial density of Mo. Next, we calculate the shock temperature $T(V)$ and the thermal energy $E_{T h}(V, T)$ along the Hugoniot via numerical integration using the known $\gamma(V)$ and $C_{V}(\Theta(V) / T)=C_{V}(V, T)$ functions. Then we obtain the thermal pressure correction $\Delta P_{T h}(V)=\mathcal{X}(V) / V^{*}\left[E_{T h}(V, T)-E_{T h}\left(V, T_{0}\right)\right]$ and the strength correction $\Delta P_{\text {str }}(V)$ given by Eq. 17 in [25]. This procedure yields the most accurate isotherm in the high compression range $\left(V / V_{0}<0.85\right)$, where the primary Mo EOS was recently calibrated [7]. In the compression range from ambient to $V / V_{0} \approx 0.85$, a blunt subtraction of $\Delta P_{s t r}(V)$ would under-estimate the isotherm pressure. To eliminate this artifact, mainly caused by poorly known $\sigma_{x}(V)$ in the range of shock compression experiments where timedependent elastic-plastic shock waves exist, we apply a cubic-spline interpolation for the strength correction using the boundary values of zero at ambient conditions and $1 \mathrm{GPa}$ at $V / N_{0} \approx 0.85$.

Table S4. Parameters used in the reduced $298 \mathrm{~K}$ isotherm from shock compression data

\begin{tabular}{|c|c|c|}
\hline Parameters & Notes & References \\
\hline$\rho_{0}: 10205 \mathrm{~kg} / \mathrm{m}^{3}$ & Density at ambient conditions & \multirow[b]{4}{*}{$\begin{array}{l}\text { Fat'yanov and } \\
\text { Asimow [7] }\end{array}$} \\
\hline $\begin{array}{l}U_{s}=5.09(2)+1.264(9) U_{p} \\
\mathrm{~km} / \mathrm{s}\end{array}$ & $\begin{array}{l}\text { Linear principal Hugoniot, where } U_{s} \text { is the shock } \\
\text { velocity and } U_{p} \text { is the particle velocity }\end{array}$ & \\
\hline$\gamma_{0}: 1.54(7)$ & Grüneisen parameter at ambient conditions & \\
\hline$C_{p}: 249.448 \mathrm{~J} / \mathrm{kg} / \mathrm{K}$ & $\begin{array}{l}\text { Heat capacity at constant pressure at ambient } \\
\text { conditions }\end{array}$ & \\
\hline $\begin{array}{l}C_{D}: 240.275 \mathrm{~J} / \mathrm{kg} / \mathrm{K} \\
C_{e l}: 7.424 \mathrm{~J} / \mathrm{kg} / \mathrm{K}\end{array}$ & $\begin{array}{l}\text { Heat capacity values at ambient conditions in a } \\
\text { combined Debye and electronic specific heat } \\
\text { model }\end{array}$ & This study \\
\hline$\Theta_{0}: 380 \mathrm{~K}$ & Debye temperature at ambient pressure & {$[6,26]$} \\
\hline $\begin{array}{l}Y\left(\sigma_{x}\right)=Y_{o}\left[\left(1+0.0070(11) \sigma_{x}\right]\right. \\
Y_{0}: 1.25(13) \mathrm{GPa}\end{array}$ & $\begin{array}{l}\text { The yield strength model for shocked Mo, } \\
\text { where } Y_{0} \text { is the yield strength at ambient } \\
\text { pressure and } \sigma_{x} \text { is the longitudinal stress under } \\
\text { shock in GPa }\end{array}$ & $\begin{array}{l}\text { This study, see Fig. } \\
\text { S4 and Fig. S5 }\end{array}$ \\
\hline
\end{tabular}

The uncertainties in pressure on the 298K-isotherm are shown in Fig. S2. In the error analysis, we made use of two important correlations between parameter uncertainties: the correlation coefficient between the uncertainties of Hugoniot slope and intercept is -1.0 and the correlation coefficient between the uncertainties of $\gamma_{0}$ and the slope of the principal Hugoniot is +0.5 . No correlation is 
assumed between the uncertainties of the strength model parameters and the thermodynamic functions. The major source of error is found to be the uncertainty in the thermal pressure correction. At $150 \mathrm{GPa}$, the uncertainty in pressure from the thermal correction accounts for $>90 \%$ of the total uncertainty in pressure. However, in the low compression range of 0-58 GPa, the uncertainties of pressure are perhaps unexpectedly large (as shown in Fig. S2) and this is due to uncertainty in the Hugoniot stress in the two-wave region and to the use of a cubic-spline interpolation to eliminate artificial kinks on the $P(V)$ curve.

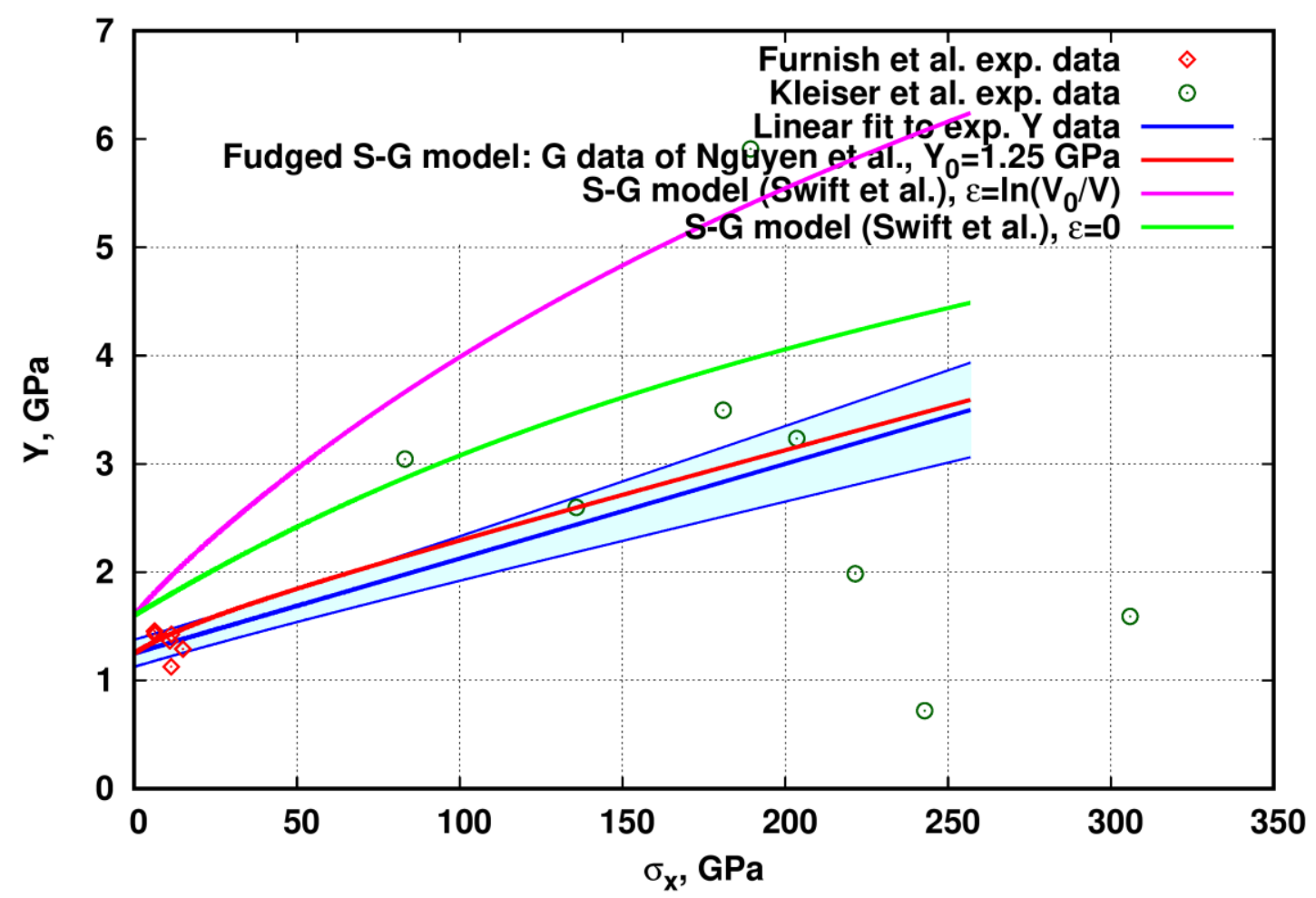

Figure S4. Experimental data [27-29] on the strength of Mo as a function of Hugoniot stress. It appears that the Steinberg-Guinan (S-G) model $[30,31]$ does not describe the experimental data of Mo very well. Here, we use a simple linear function (blue line, with 1- $\sigma$ uncertainty shown as light-blue shaded area) to represent the strength of Mo in 0-260 GPa: $Y\left(\sigma_{x}\right)=Y_{0}^{*}\left[1+0.0070(11) \sigma_{x}\right]$, with $Y_{0}=1.25(13) \mathrm{GPa}$. An empirical adjustment to the S-G model using the shear modulus data of [29] can yield a strength curve effectively identical to the adopted linear model (see Fig. S5). 


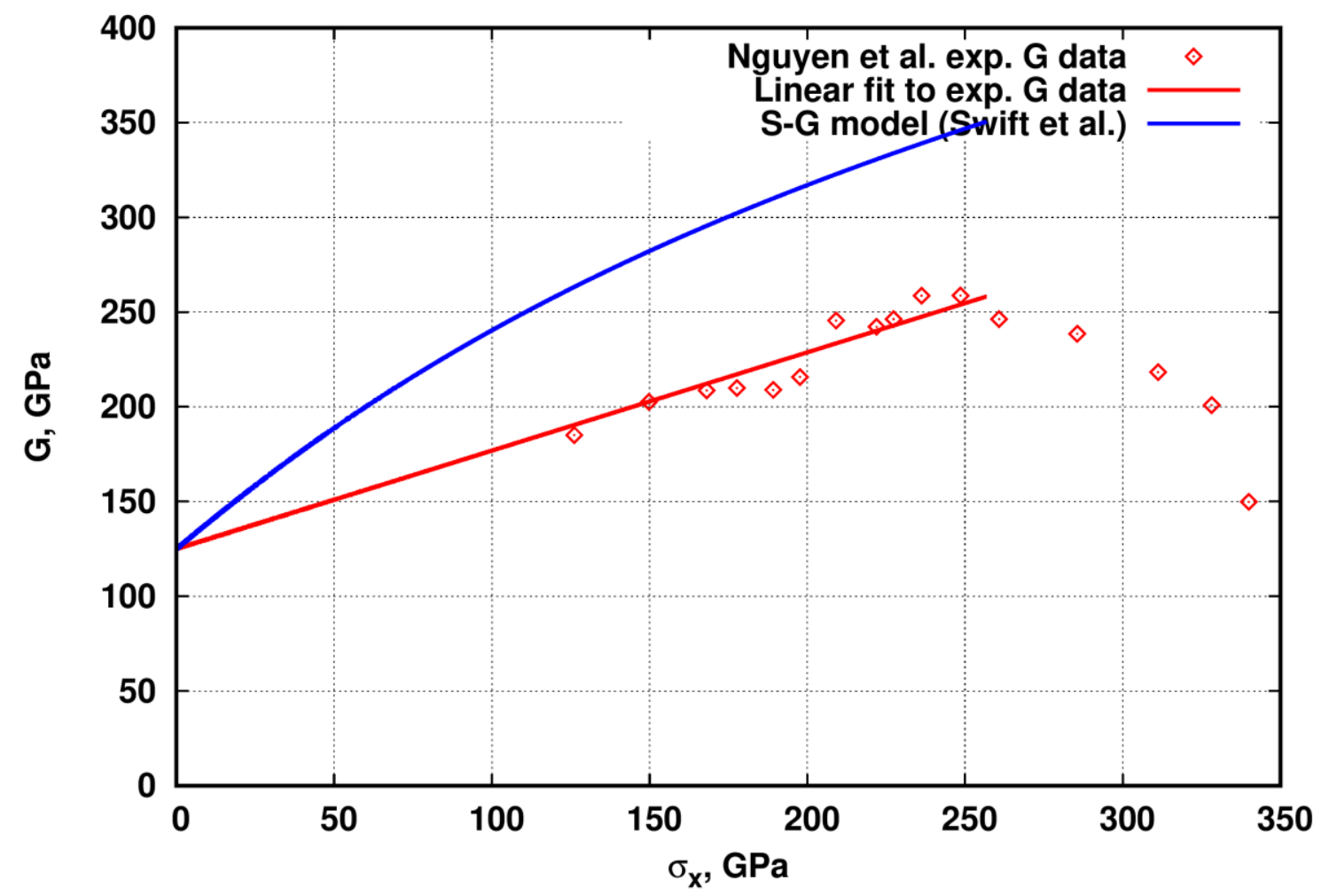

Figure S5. Experimental data [29] for the shear modulus $(G)$ of shock-compressed Mo. A linear function, $G=G_{0}\left[1+0.00415(10) \sigma_{x}\right]$ with $G_{0}$ of $125 \mathrm{GPa}$, provides a better fit than the S-G model [30, 31]. Using this linear model of $G$ in a modified $S-G$ model produces a strength model similar to an empirical linear strength model (see Fig. S4). 


\section{References}

[1] Wu, C, Thermal subcomponent analysis on the applicability of reduced-shock-wave data. manuscript in preparation.

[2] Mao, HK, Bell, PM, Shaner, JW, et al., Specific volume measurements of $\mathrm{Cu}, \mathrm{Mo}, \mathrm{Pd}$, and $\mathrm{Ag}$ and calibration of the ruby R1 fluorescence pressure gauge from 0.06 to 1 Mbar. J. Appl. Phys., 1978. 49(6):3276-3283.

[3] Carter, WJ, Marsh, SP, Fritz, JN, et al., The equation of state of selected materials for high pressure references. NBS Spec. Publ. , 1971. 36147.

[4] Hixson, RS and Fritz, JN, Shock compression of tungsten and molybdenum. J. Appl. Phys., 1992. 71(4):1721-1728.

[5] Wang, Y, Ahuja, R, and Johansson, B, Reduction of shock-wave data with mean-field potential approach. J. Appl. Phys., 2002. 92(11):6616-6620.

[6] Al'tshuler, LV, Brusnikin, SE, and Kuz'menkov, EA, Isotherms and Grüneisen functions for 25 metals. JAMTP, 1987. 28(1):129-141.

[7] Fat'yanov, OV and Asimow, PD, Equation of state of Mo from shock compression experiments on preheated samples. J. Appl. Phys., 2017. 121(11):115904.

[8] Fratanduono, DE, Smith, RF, Ali, SJ, et al., Probing the solid phase of noble metal copper at terapascal conditions. Phys. Rev. Lett., 2020. 124(1):015701.

[9] Kraus, RG, Davis, JP, Seagle, CT, et al., Dynamic compression of copper to over $450 \mathrm{GPa}$ : A highpressure standard. Phys. Rev. B, 2016. 93(13):134105.

[10] Chijioke, AD, Nellis, WJ, and Silvera, IF, High-pressure equations of state of $\mathrm{Al}, \mathrm{Cu}, \mathrm{Ta}$, and W. J. Appl. Phys., 2005. 98(7):073526.

[11] Dewaele, A, Torrent, M, Loubeyre, P, et al., Compression curves of transition metals in the Mbar range: Experiments and projector augmented-wave calculations. Phys. Rev. B, 2008. 78(10):104102.

[12] Dewaele, A, Loubeyre, P, and Mezouar, M, Equations of state of six metals above $94 \mathrm{GPa}$. Phys. Rev. B, 2004. 70(9):094112.

[13] Zha, C-S, Mao, H-k, and Hemley, RJ, Elasticity of MgO and a primary pressure scale to $55 \mathrm{GPa}$. Proc. Natl. Acad. Sci., 2000. 97(25):13494-13499.

[14] Occelli, F, Loubeyre, P, and LeToullec, R, Properties of diamond under hydrostatic pressures up to 140 GPa. Nat. Mat., 2003. 2(3):151-154.

[15] Mao, $\mathrm{HK}, \mathrm{Xu}, \mathrm{J}$, and Bell, PM, Calibration of the ruby pressure gauge to 800 kbar under quasihydrostatic conditions. J. Geophys. Res., 1986. 91(B5):4673-4676.

[16] Aleksandrov, IV, Goncharov, AF, Zisman, AN, et al., Diamond at high pressures - Raman scattering of light, equation of state, and a high pressure scale. Sov. Phys. JETP, 1987. 66:384390.

[17] Kunc, K, Loa, I, and Syassen, K, Diamond under pressure: Ab-initio calculations of the equation of state and optical phonon frequency revisited. High Pressure Res., 2004. 24(1):101-110.

[18] Holzapfel, WB, Progress in the realization of a practical pressure scale for the range 1-300 GPa. High Pressure Res., 2005. 25(2):87-99.

[19] Chijioke, AD, Nellis, WJ, Soldatov, A, et al., The ruby pressure standard to 150GPa. J. Appl. Phys., 2005. 98(11):114905.

[20] Dorogokupets, $\mathrm{PI}$ and Oganov, AR, Ruby, metals, and $\mathrm{MgO}$ as alternative pressure scales: $A$ semiempirical description of shock-wave, ultrasonic, $x$-ray, and thermochemical data at high temperatures and pressures. Phys. Rev. B, 2007. 75(2):024115. 
[21] Jacobsen, SD, Holl, CM, Adams, KA, et al., Compression of single-crystal magnesium oxide to 118 GPa and a ruby pressure gauge for helium pressure media. Am. Min., 2008. 93(11-12):18231828.

[22] Syassen, K, Ruby under pressure. High Pressure Res., 2008. 28(2):75-126.

[23] Holzapfel, WB, Equations of state for $\mathrm{Cu}, \mathrm{Ag}$, and $\mathrm{Au}$ and problems with shock wave reduced isotherms. High Pressure Res., 2010. 30(3):372-394.

[24] Sokolova, TS, Dorogokupets, PI, and Litasov, KD, Self-consistent pressure scales based on the equations of state for ruby, diamond, $\mathrm{MgO}, \mathrm{B2}-\mathrm{NaCl}$, as well as $\mathrm{Au}, \mathrm{Pt}$, and other metals to 4 Mbar and 3000 K. Russian Geology and Geophysics, 2013. 54(2):181-199.

[25] Morris, CE and Fritz, JN, Relation of the "solid Hugoniot" to the "fluid Hugoniot" for aluminum and coppera). J. Appl. Phys., 1980. 51(2):1244-1246.

[26] Bashir, J, Butt, NM, Nasir Khan, M, et al., Determination of the Debye-Waller factor of molybdenum by powder neutron diffraction. J. Appl. Crystallogr., 1992. 25(6):797-799.

[27] Furnish, MD, Chhabildas, LC, Steinberg, DJ, et al., Dynamic behavior of fully dense molybdenum, in Shock Compression of Condensed Matter-1991, S.C. Schmidt, et al., Editors. 1992, Elsevier: Amsterdam. p. 419-422.

[28] Kleiser, GJ, Chhabildas, LC, Reinhart, WD, et al., Using time-resolved wave profile measurements to determine elusive phase transitions in molybdenum. Procedia Engineering, 2013. 58:617-623.

[29] Nguyen, JH, Akin, MC, Chau, R, et al., Molybdenum sound velocity and shear modulus softening under shock compression. Phys. Rev. B, 2014. 89(17):174109.

[30] Swift, DC, Seifter, A, Holtkamp, DB, et al., Shock and release temperatures in molybdenum: Experiment and theory. Phys. Rev. B, 2007. 76(5):054122.

[31] Alexander, CS, Ding, JL, and Asay, JR, Experimental characterization and constitutive modeling of the mechanical behavior of molybdenum under electromagnetically applied compression-shear ramp loading. J. Appl. Phys., 2016. 119(10):105901. 